\title{
Effective Triple-Negative Breast Cancer Targeted Treatment Using iRGD-Modified RBC Membrane-Camouflaged Nanoparticles
}

\author{
Jingbin Huang* \\ Wenjing Lai* \\ Qing Wang \\ Qin Tang \\ Changpeng $\mathrm{Hu}$ \\ Min Zhou \\ Fengling Wang \\ Dandan Xie \\ Qian Zhang \\ Wuyi Liu \\ Zhe Zhang \\ Rong Zhang
}

Department of Pharmacy, The Second Affiliated Hospital of Army Medical University, ChongQing, People's Republic of China

*These authors contributed equally to this work

\begin{abstract}
Introduction: Triple-negative breast cancer (TNBC) has the high degree of malignancy and aggressiveness. There is no targeted therapy drug. Many studies have shown that RBC membrane-coated nanoparticles achieve biological camouflage. In addition, the RGD module in the iRGD mediates the penetration of the vector across the tumor blood vessels to the tumor tissue space. Therefore, we developed iRGD-RM-(DOX/MSNs) by preparing MSNs loaded with doxorubicin as the core, and coating the surface of the MSNs with iRGDmodified RBC membranes.
\end{abstract}

Methods: iRGD-RM-(DOX/MSNs) were fabricated using physical extrusion. In addition, their physical and chemical characterization, hemolytic properties, in vivo acute toxicity and inflammatory response, in vitro and in vivo safety, and qualitative and quantitative cellular uptake by RAW 264.7 cells and MDA-MB-231 cells were evaluated and compared. Furthermore, we examined the antitumor efficacy of iRGD-RM-(DOX/MSN) nanoparticles in vitro and in vivo.

Results: iRGD-RM-(DOX/MSNs) have reasonable physical and chemical properties. iRGDRM-(DOX/MSNs) escaped the phagocytosis of immune cells and achieved efficient targeting of nanoparticles at the tumor site. The nanoparticles showed excellent antitumor effects in vivo and in vitro.

Conclusion: In this study, we successfully developed biomimetic iRGD-RM-(DOX/MSNs) that could effectively target tumors and provide a promising strategy for the effective treatment of TNBC.

Keywords: RBC membrane, iRGD peptide, biological camouflage, targeted treatment, Triple-negative breast cancer

\section{Introduction}

Breast cancer is estimated to account for $30 \%$ of the estimated cancer diagnoses among women in America in 2021. ${ }^{1}$ Breast cancer is one of the most severe diseases worldwide and poses a serious threat to the health of women. Triple-negative breast cancer (TNBC) has the highest degree of malignancy and is the most aggressiveness breast cancer subtype, with early metastasis, recurrence and poor prognosis. There is no clinical targeted therapy drug for TNBC due to the lack of estrogen receptor, progesterone receptor and Her-2 receptor on the cell surface. ${ }^{2-5}$ At present, surgery, chemotherapy, radiation therapy, immunotherapy and hormone therapy have been utilized in the treatment of breast cancer. Among them, standard chemotherapy and radiation are the main therapeutic options for patients with TNBC. However, such
Correspondence: Rong Zhang; Zhe

Zhang

Email xqpharmacylab@I26.com;

I5086988023@I63.com 
treatments have adverse side effects and usually fail due to nontargeted delivery and the acquired resistance to chemotherapy drugs in vivo, resulting in aggressive metastatic relapse and short overall survival. ${ }^{6-10}$ Therefore, it is necessary to develop a new strategy to effectively target breast cancer cells to improve the effectiveness of chemotherapy drugs. An ideal targeting strategy should also possess features of high efficiency in reaching target sites and strong penetration of target cells.

Recently, some tumor-penetrating peptides (TPPs) that possess the ability to promote the penetration of nanoparticles and molecules into tumor cells have been developed. $^{11,12}$ For instance, iRGD, as a cyclic tumorpenetrating peptide, is composed of an RGD module and an overlapping $\mathrm{C}$-end $\mathrm{R}$ module connected by a disulfide bond. Its tumor-specific targeting and penetrating abilities are significantly superior to those of the RGD peptide. ${ }^{13-15}$ The RGD module in the iRGD sequence has been demonstrated to bind to $\alpha v \beta 3$ or $\alpha v \beta 5$ integrin, which are specifically expressed in tumor vascular endothelial cells. In addition, it mediates the penetration of the vector across the tumor blood vessels to the tumor tissue space. Then, iRGD is cleaved by a cell surface-associated protease to expose the $\mathrm{C}$-end $\mathrm{R}$ motif and allow for specific binding to neuropilin-1 (NRP-1), which is highly expressed on the surface of tumor cells, to trigger its interalization. ${ }^{13,16,17}$ It has been reported that iRGD-modified drugs or drug delivery systems can achieve deep penetration of tumors and significantly enhance the therapeutic effect of antitumor drugs. ${ }^{18,19}$ Furthermore, the vascular endothelium of triple-negative breast cancer has high expression of $\alpha v \beta 3$ and $\alpha v \beta 5$ integrin, and its tumor cell surface has high levels of NRP-1 expression. ${ }^{6,20}$ Therefore, iRGD is expected to be an effective ligand for specifically targeting TNBC in the absence of the multiple surface receptors for TNBC.

The liver, spleen and other tissues and organs have accumulated considerable amounts of nanoparticles from passive or active targeting drug delivery systems. Therefore, the immune recognition and elimination of nanoparticles is the main obstacle achieving tumorspecific targeting with nanoparticles. To overcome the immune recognition of nanoparticles, one strategy is to modify the surface of nanoparticles with a hydrophilic polymer, the most commonly used being polyethylene glycol (PEG). PEG-modified nanoparticles can effectively reduce phagocytosis through the mononuclear phagocyte system (MPS) to achieve long circulation throughout the body. ${ }^{21-23}$ However, PEG-modified nanoparticles can also stimulate the immune system after multiple administrations, causing subsequent administration of nanoparticles to be recognized and swallowed by the immune system; therefore, immune escape cannot be achieved. ${ }^{24}$ In recent years, studies have focused on the new field of biological camouflage nanocarriers, and one of the effective methods is using cell membrane-coated nanoparticles to achieve biological camouflage. ${ }^{25-27}$ The proteins and glycosyl groups on the surface of the membrane endow the nanoparticles with prolonged systematic residence, reduced the uptake by the reticuloendothelial system (RES), and moderate immune recognition, thereby reducing immune elimination and improving nanoparticle or drug accumulation at tumor sites. ${ }^{28-30}$ Ligands targeting tumor site-modified cell membranes are likely to further improve the targeting efficiency. Herein, we modified the targeting peptide iRGD on the surface of the red blood cell membrane to achieve efficient targeting of tumor cells.

On the other hand, the biocamouflage drug-loading system not only enables the carrier to have better biocompatibility, but also has a higher drug-carrying capacity. The cell membrane coating strategy has been widely used in nanostructures, such as silica nanoparticles, PLGA nanoparticles, nanogels and gold nanoparticles. ${ }^{31-34}$ Here, we chose mesoporous silica nanoparticles (MSNs), which are inert porous nanomaterials, that are nontoxic to the body with large pore capacity, that can adsorb drug molecules in the pores to achieve high drug loading. ${ }^{35,36}$

In this study, we prepared a tumor-targeting ligand iRGD-modified RBC membrane for antitumor drug delivery. Specifically, we chose doxorubicin (DOX), which is a classic clinical chemotherapy drug, and loaded it into mesoporous silica nanoparticles (MSNs) to achieve high drug loading. Finally, the iRGDmodified RBC membrane was coated on the surface of MSNs to form iRGD-RM-(DOX/MSN). In vitro studies confirmed the favorable stability and blood compatibility, and the low cytotoxicity of iRGD-RM-MSNs. In vivo safety evaluations showed a low immune response and low systemic toxicity of iRGD-RM-(DOX/MSNs) and RM-(DOX/MSNs) compared with DOX/MSN. In the anti-subcutaneous orthotopic breast cancer transplantation tumor model, iRGD-RM-(DOX/MSNs) showed significant tumor-targeting capability and enhanced antitumor efficacy. 


\section{Materials and Methods}

RAW 264.7 and MDA-MB-23I Cells

\section{Culture Methods}

RAW 264.7 and MDA-MB-231 cells were obtained from the Cell Culture Center of the Shanghai Institutes for Biological Sciences of the Chinese Academy of Sciences (Shanghai, China) and cultured in Dulbecco's modified Eagle medium (DMEM) supplemented with 10\% fetal bovine serum (FBS). Cells were cultured in a cell culture incubator at $37{ }^{\circ} \mathrm{C}$ with $5 \%$ carbon dioxide.

\section{Preparation and Characterization of iRGD-RM-(DOX/MSNs)}

\section{Extraction of Red Blood Cell (RBC) Membrane}

The $\mathrm{RBC}$ membranes were prepared according to a previous study. ${ }^{37,38}$ First, whole blood was collected from healthy male Sprague-Dawley rats into anticoagulant tubes. Then, fresh whole blood was centrifuged at $4000 \mathrm{rpm}$ for $5 \mathrm{~min}$ at $4{ }^{\circ} \mathrm{C}$ to remove the plasma and buffy coat and washed three times with cold PBS to obtain RBCs. The as-washed RBCs were resuspended in $0.25 \times$ PBS (volume ratio of RBCs to PBS was 1:15), kept in an ice bath for $30 \mathrm{~min}$ and centrifuged at $800 \mathrm{x} \mathrm{g}$ for $5 \mathrm{~min}$ at $4{ }^{\circ} \mathrm{C}$. Then, the pellet was collected and washed with $0.25 \times$ PBS until the supernatant was colorless. Finally, the RBC membranes (light pink pellet) were collected and stored at $4{ }^{\circ} \mathrm{C}$.

Synthesis and Characterization of DSPE-PEG $2 k^{-i R G D}$ DSPE-PEG $_{2 \mathrm{k}}$-iRGD was synthesized according to a previous report. ${ }^{39}$ Briefly, $20.4 \mathrm{mg}$ iRGD and $50 \mathrm{mg}$ DSPE-PEG $_{2 \mathrm{k}}$-NHS (1.2:1 mol ratio) were dissolved in $10 \mathrm{~mL}$ of DMSO, after which $10 \mu \mathrm{L}$ of triethylamine was added, and the mixture was stirred at $37{ }^{\circ} \mathrm{C}$ for 12 $\mathrm{h}$. Then, the reaction mixture was dialyzed in deionized water with a dialysis membrane $(\mathrm{MWCO}=3400 \mathrm{Da})$ to remove solvents and excess iRGD and then lyophilized. The chemical structure and molecular weight of DSPE$\mathrm{PEG}_{2 \mathrm{k}}$-iRGD were characterized by ${ }^{1} \mathrm{H}-\mathrm{NMR}$ and MALDI-TOF, respectively.

Preparation and Characterization of the iRGD-Modified RBC Membrane (iRGD-RM)

For the iRGD-modified RBC membrane (iRGD-RM) preparation, the RBC membrane (derived from $200 \mu \mathrm{L}$ of whole blood) was suspended in $820 \mu \mathrm{L}$ of PBS, and 180 $\mu \mathrm{L}$ of DSPE-PEG $\mathrm{2k}_{\mathrm{k}}-\mathrm{iRGD}(100 \mu \mathrm{g} / \mathrm{mL})$ was added. Then, the mixture was stirred at $37{ }^{\circ} \mathrm{C}$ for one hour. After that, free DSPE-PEG $2 \mathrm{k}-\mathrm{iRGD}$ was washed away by centrifugation at $800 \mathrm{x} \mathrm{g}$ for 5 minutes, and the pellets were washed three times with PBS. The obtained iRGDRMs were resuspended in $0.5 \mathrm{~mL}$ of $0.25 \times \mathrm{PBS}$ for further use. Then, the obtained RM or iRGD-RM was sonicated for $5 \mathrm{~min}$ and extruded through $400 \mathrm{~nm}$ and $200 \mathrm{~nm}$ polycarbonate porous membranes with a liposome extruder. The hydrodynamic diameters and zeta potentials of the newly membranes were characterized by a Malvern Instrument (ZS90, Malvern, UK).

Synthesis and Characterization of Mesoporous Silica Nanoparticles (MSNs)

MSNs were synthesized from TEOS in the presence of CTAB according to a previous study, with some modifications. CTAB $(0.1 \mathrm{~g}), 0.35 \mathrm{~mL}$ of $\mathrm{NaOH}(2 \mathrm{M})$ and $50 \mathrm{~mL}$ of deionized water were added into a $250 \mathrm{~mL}$ round-bottom flask and ultrasonically blended. The mixture was preheated to $80{ }^{\circ} \mathrm{C}$ for $15 \mathrm{~min}$. Then, $0.55 \mathrm{~mL}$ of TEOS was added, followed by the addition of $0.5 \mathrm{~mL}$ of ethyl acetate when the solution began to turn white. The solution was magnetically stirred for an additional 2 hours at $80{ }^{\circ} \mathrm{C}$. The obtained nanoparticles were first centrifuged at $5000 \mathrm{rpm}$ for $5 \mathrm{~min}$ to remove large aggregates, and the supernatant was collected. Subsequently, the mixture was centrifuged and alternatively washed with water and ethanol several times. Thereafter, the nanoparticles were refluxed in an ethanol/hydrochloric acid solution $(\mathrm{v} / \mathrm{v}=9: 1)$ for 12 hours at $80{ }^{\circ} \mathrm{C}$. Then, the nanoparticles were repeatedly washed at least three times with water and ethanol. Finally, the obtained white precipitates were resuspended in water, the $\mathrm{pH}$ value was adjusted to 7.0, and the precipitates were lyophilized. The product was characterized by N2 adsorption-desorption isotherm experiments.

\section{Fabrication and Characterization of iRGD-RM- (DOX/MSNs)}

Thirty-six milligrams of MSNs were mixed with $4 \mathrm{mg}$ of DOX in $4 \mathrm{~mL}$ of deionized water, followed by stirring for $24 \mathrm{~h}$ in the dark at room temperature. The mixture was washed three times in deionized water until a clear supernatant was obtained. For membrane-coated nanoparticle preparation, DOX/MSNs (containing $1 \mathrm{mg}$ of MSNs) were mixed with $400 \mathrm{~nm}$ iRGD-RM (obtained from 100 $\mu \mathrm{L}$ of blood) and extruded through $400 \mathrm{~nm}$ polycarbonate porous membranes several times. Subsequently, the nanoparticles were centrifuged at $10,000 \mathrm{rpm}$ for $5 \mathrm{~min}$ and washed with $0.25 \times$ PBS buffer three times. Then, the obtained iRGD-RM-(DOX/MSN) nanoparticles were 
lyophilized. RM-(DOX/MSN) nanoparticles were prepared using the same method except that unmodified RM was added.

The physicochemical properties of the iRGD-RM -(DOX/MSN) nanoparticles were characterized with the size, zeta potential, morphology, stability test, adsorption with protein, drug loading and encapsulation efficiency. The size and zeta potential of the nanoparticles were measured by a Malvern Instrument (Nano-ZS 90, Malvern, UK). Transmission electron microscopy (TEM) was used to visualize the morphology of the nanoparticles under a TECNAI-10 microscope (Philips, Netherlands). Membrane-coated nanoparticles were stained with uranyl acetate before visualization. The drug loading and encapsulation efficiency of DOX were determined by an indirect method. Briefly, the supernatant was collected, and the unloaded DOX was detected by HPLC (Agilent 1200, USA) through a reversed-phase C-18 column (Phenomenex Luna ${ }_{(2)}, 250 \times 4.6 \mathrm{~mm}, 5 \mu \mathrm{m}$ ). For HPLC analysis, the mobile phase consisted of methanol and $0.15 \% \quad \mathrm{H}_{3} \mathrm{PO}_{4}$ water $(52 / 48, \mathrm{v} / \mathrm{v})$, the flow rate was $1 \mathrm{~mL} / \mathrm{min}$, the detection wavelength was $233 \mathrm{~nm}$, the column temperature was $40^{\circ} \mathrm{C}$, the injection volume was $10 \mu \mathrm{L}$, and samples were filtered in a $0.45 \mathrm{~mm}$ pore filter (Millipore, USA) before injection.

\section{In vitro Drug Release of iRGD-RM- (DOX/MSNs)}

To evaluate the in vitro drug release behavior of drugloaded nanoparticles, $18 \mathrm{mg}$ of DOX-loaded nanoparticles were suspended in $72 \mathrm{~mL}$ of $\mathrm{pH} 5.0$ or 7.4 PBS. Subsequently, $2 \mathrm{~mL}$ of the suspension was drawn into a series of EP tubes, and maintained at $37{ }^{\circ} \mathrm{C}$ with a shaking speed of $100 \mathrm{rpm}$ (Thermo, MaxQ 4000). The samples were moved at predetermined time intervals and centrifuged at 13,000 rpm for $5 \mathrm{~min}$. The supernatant was collected, and the DOX concentration was determined by a fluorescence method $(\mathrm{Ex} / \mathrm{Em}=469 / 593 \mathrm{~nm}, \mathrm{Ex} / \mathrm{Em}$ shift $=5 \mathrm{~nm}$, PMT voltage $=500 \mathrm{~V}$ ). The released amount of DOX was calculated as follows: Drug release = $\left(\mathrm{W}_{\mathrm{i}} / \mathrm{W}_{\text {whole }}\right) \times 100 \%$, where $\mathrm{W}_{\mathrm{i}}$ is the amount of accumulatively released $\mathrm{DOX}$ and $\mathrm{W}_{\text {whole }}$ is the whole amount of DOX.

\section{Hemolysis Assay of iRGD-RM-MSNs}

The hemolysis assay was performed according to a previous study. RBCs were obtained according to
"Extraction of Red Blood Cell (RBC) Membrane". The cells were diluted to $1 / 10$ of their volume with PBS, and then $0.3 \mathrm{~mL}$ of the RBCs solution was added into a $1.5 \mathrm{~mL}$ centrifuge tube, followed by centrifuging at $2000 \mathrm{x}$ g for 5 min to remove the residual PBS. Next, different concentrations of MSN, RM-MSN, iRGD-RM-MSN in $1.0 \mathrm{~mL}$ of PBS (7.82-1000 g/mL) were added to the RBCs and gently mixed on a horizontal shaker (MaxQ4000, Thermo Scientific, USA) at a speed of $100 \mathrm{rpm}$ at $37^{\circ} \mathrm{C}$. The groups treated with $1 \times$ PBS or deionized water were used as negative and positive controls, respectively. Two hours later, the samples were centrifuged at $2000 \mathrm{x}$ g for 5 min, and the absorbance of the supernatants at $541 \mathrm{~nm}$ was measured by a microplate reader (M MLTISCAN GO, Thermo, USA). Hemolysis percentages of the formulations were calculated with the following equation: Hemolysis $\%$ $=\left(\mathrm{A}_{\text {sample }}-\mathrm{A}_{(-) \text {control }}\right) /\left(\mathrm{A}_{(+) \text {control }}-\mathrm{A}_{(-) \text {control }}\right) . \mathrm{A}$ is the absorbance value.

\section{In vivo Immunogenicity and Acute Toxicity of DOX-Loaded Nanoparticles}

Healthy female mice were randomly divided into four groups (n = 4): (1) PBS; (2) DOX/MSN; (3) RM-(DOX/ MSN); and (4) iRGD-RM-(DOX/MSN). The mice were administrated different formulations via tail vein at an equivalent DOX dosage of $30 \mathrm{mg} / \mathrm{kg}$ (threefold higher than the therapeutic dosage). Blood samples were collected from the orbital venous plexus at 12 and 24 hours postinjection. For immunogenicity analysis, blood samples were collected and centrifuged at $8000 \mathrm{rpm}$ to collect serum. Subsequently, cytokines (IL-1 $\beta$, IL-6 and TNF- $\alpha$ ) were detected using ELISA kits according to the manufacturer's instructions. For blood analysis, blood samples were collected into heparin, and red blood cells (RBCs), white blood cells (WBCs), blood platelets (PLTs), the mean platelet volume (MPV), the mean corpuscular volume (MCV) and hemoglobin (HGB) were analyzed.

\section{In vitro Cellular Uptake of DOX-Loaded Nanoparticles}

The cellular uptake of different DOX-loaded nanoparticles was examined by confocal laser microscopy and flow cytometry. Before confocal laser microscopy imaging or flow cytometry analysis, RAW-264.7 and MDA-MB-231 cells were grown on glass coverslips in 24-well plates (or cultured in 6-well culture plates for flow cytometry analysis) overnight. Then the cells were incubated with free 
DOX, DOX/MSNs, RM-(DOX/MSNs) or iRGD-RM -(DOX/MSNs) at an equivalent DOX concentration of 10 $\mu \mathrm{g} / \mathrm{mL}$ for 0.5 hours or 1 hour. For confocal laser microscopy imaging, the cells were fixed with immunostaining fixative for $15 \mathrm{~min}$, permeabilized with $0.1 \%$ Triton X-100 for $5 \mathrm{~min}$, incubated with phalloidin-F488 for $30 \mathrm{~min}$ and then counterstained with DAPI for $5 \mathrm{~min}$ at $25^{\circ} \mathrm{C}$. Images were taken with an LSM780NLO confocal laser scanning microscope (Zeiss, Germany). For flow cytometry analysis, the cells were collected after the administration of each treatment, centrifuged at $1500 \mathrm{rpm}$ for $5 \mathrm{~min}$, and washed three times with PBS ( $\mathrm{pH}$ 7.4). After that, cells were suspended in $300 \mu \mathrm{L}$ of PBS and analyzed by flow cytometry (Accuri C6, BD, American). For the blocking test, cells were preincubated with free iRGD for $30 \mathrm{~min}$, after which the iRGD was removed, and the cells were incubated with iRGD-RM-(DOX/MSNs).

\section{In vitro Cytotoxicity of DOX-Loaded Nanoparticles}

3-(4,5-dimethylthiazol-2-yl)-2,5-diphenyltetrazolium bromide (MTT) Assay

The cytotoxicity of different DOX-loaded nanoparticles treated on MDA-MB-231 cells was measured using the MTT assay. Briefly, MDA-MB-231 cells were seeded in 96-well plates at a density of 4000 cells per well, and incubated overnight. Then, the medium was replaced with fresh medium containing empty nanoparticles (MSNs, RM-MSNs and iRGD-RM-MSNs) or a series of concentrations of free DOX, DOX-loaded MSNs (DOX/ MSNs), RBC membrane-coated DOX/MSNs (RM-DOX /MSNs), and iRGD-modified RM-DOX/MSNs (iRGDRM-(DOX/MSNs)). After 48 hours of incubation, the cells were washed with PBS, and $100 \mu \mathrm{L}$ of fresh medium was added to each well. Subsequently, $20 \mu \mathrm{L}$ of $5 \mathrm{mg} / \mathrm{mL}$ MTT solution was added to each well and incubated for another $4 \mathrm{~h}$. Then, removed the medium containing MTT removed, $150 \mu \mathrm{L}$ of DMSO was added to each well, and the absorbance at $490 \mathrm{~nm}$ was measured using a microplate reader (M MLTISCAN GO, Thermo, USA).

\section{Apoptosis Assay by Flow Cytometry}

Apoptosis assays were performed to using an apoptosis staining kit as described below. Briefly, MDA-MB-231 cells were seeded in six-well plates at a density of $1 \times 10^{5}$ cells/well. The cells were assigned to six groups: (i) control group; (ii) DOX group; (iii) DOX-loaded MSN nanoparticles (DOX/MSN); (iv) RM-(DOX/MSN); (v) iRGD-
RM-(DOX/MSN); and (vi) DSPE-PEG-iRGD + iRGDRM-(DOX/MSN) ((DSPE-PEG-iRGD has tenfold higher iRGD than iRGD-RM-(DOX/MSN) and was added 30 min prior to iRGD-RM-(DOX/MSN)). Each group contained $500 \mathrm{ng} / \mathrm{mL}$ of DOX. After forty-eight hours of treatment, cells were trypsinized, collected, and then washed twice with PBS. Cells were resuspended in 100 $\mu \mathrm{L}$ of binding buffer with $2.5 \mu \mathrm{L}$ of Annexin V-FITC and $2.5 \mu \mathrm{L}$ of 7-AAD (APC Annexin V Apoptosis Detection Kit with 7-AAD, BioLegend). After incubation for 15 min in the dark at $25{ }^{\circ} \mathrm{C}$, an additional $200 \mu \mathrm{L}$ of binding buffer was added to each tube. Finally, the cells in each tube were analyzed by flow cytometry (FACSAria III, BD).

\section{The Animal Studies}

All procedures involving animals were carried out in accordance with the National Institutes of Health Guidelines for the Care and Use of Laboratory Animals. At the same time, it had been approved by the Animal Ethical and Experimental Committee of Army Military Medical University. Female nude mice ( $15 \pm 2$ g), female C57BL/6 mice (20 $\pm 2 \mathrm{~g})$ and female Sprague-Dawley rats $(180 \pm 20 \mathrm{~g})$ were supplied by the Laboratory Animal Center of the Army Military Medical University. Animals were housed in cages with six mice in each cage and given free access to food and water.

\section{In vivo Antitumor Activity of DOX-Loaded Nanoparticles}

The therapeutic effects of the drug-loaded nanoparticles on breast cancer models in nude mice were determined. An orthotopic breast cancer model was constructed by orthotopic injection of $1 \times 10^{6}$ MDA-MB-231 cells under the left breast fat pad of nude mice. Tumor-bearing mice were randomly assigned to five groups (six mice per group): (i) saline; (ii) DOX; (iii) DOX/MSN; (iv) RM-(DOX/ MSN); and (v) iRGD- RM-(DOX/MSN). When the tumor volume reached $50 \mathrm{~mm}^{3}$, the treatments were administered via tail vein every three days, and the equivalent dose of DOX per formulation was $10 \mathrm{mg} / \mathrm{kg}$. Mice were measured for tumor volume with a Vernier caliper. The formula for calculating the tumor volume is as follows: $\mathrm{V}=0.5 \times$ length $\times$ wide. $^{2}$ Ten days after the first treatment, mice were sacrificed, and the tumors were weighted. The tumor growth inhibition rate was calculated, and the heart, 
liver, spleen, lung, and kidney were collected for toxicity evaluation.

\section{In vivo Biosafety Evaluation of DOX-Loaded Nanoparticles}

The tissues (heart, liver, spleen, lung and kidney) were fixed in $4 \%$ paraformaldehyde, dehydrated and then embedded in paraffin. The tissue-embedded paraffin blocks were then cut into $4-\mu \mathrm{m}$ slices and set onto polylysine-charged glass slides. The sections were deparaffinized, rehydrated through a graded alcohol series and then subjected to H\&E staining.

\section{Statistical Analysis}

Data are shown as means \pm standard deviation (SD). Statistical analysis was determined using one-way analysis of variance. A $\mathrm{P}$ value less than 0.05 was considered statistically significant $\left({ }^{*} p<0.05, \quad{ }^{* *} p<0.01\right.$, and $* * * p<0.001)$.

\section{Results}

\section{Preparation and Characterization of iRGD-RM-(DOX/MSN) Nanoparticles}

To synthesize iRGD-RM-(DOX/MSNs) that possess tumor targeting and immune escape capabilities, MSNs loaded with doxorubicin were prepared. Second, the iRGD peptide-modified RBC membrane were prepared. Considering the iRGD peptide could be better to modified insert the cell membrane, DSPE-PEG would be linked to iRGD. Then, the iRGD peptide-modified RBC membranes were coated on DOX/MSNs to endow the nanoparticles with tumor-targeting and immune escape abilities. The fabrication of TNBC-targeted biological camouflage iRGD-RM -(DOX/MSNs) is schematically illustrated in Figure 1.

\section{Preparation and Characterization of MSNs}

The biological camouflage drug delivery system not only requires the carrier to have good biocompatibility, but also needs to have high drug loading capacity. MSNs are inert, porous nanomaterials that are nontoxic and have large pore capacity. ${ }^{40,41}$ According to the method of a previous report, nanoparticles with pores were synthesized to deliver high doses of doxorubicin. ${ }^{42}$ First, MSNs were prepared and characterized. The diameter of the MSNs was $\sim 138.3 \pm 2.8 \mathrm{~nm}$ with a narrow PDI range $(0.075 \pm 0.010)$, which suggested that the MSNs had a good monopolymerization dispersion (Figure 2A). The transmission electron micrograph showed that MSNs had a well-formed spherical shape (Figure 2E and F). The zeta potential of MSNs was $-25.2 \pm 1.4 \mathrm{mV}$, and such a negative surface charge was necessary for RBC membrane coating (Figure 2B). $\mathrm{N}_{2}$ adsorption-desorption isotherm experiments showed that the adsorbed volume and pore diameter of MSNs reached $700 \mathrm{~cm}^{3} / \mathrm{g}$ and $3.9 \mathrm{~nm}$, respectively (Figure 2C and D). These results suggest that the prepared MSNs had the potential for loading a high concentration of drug.

\section{Preparation and Characterization of iRGD-Modified RBC-Coated MSNs}

As illustrated in Figure 1B, to prepare the iRGD-modified RBC membrane (iRGD-RM), the RBC membrane was derived from RBCs and further incorporated the targeting peptide- iRGD on the surface of the membranes; this peptide binds to integrin $3(\alpha v \beta 3)$ or integrin $5(\alpha v \beta 5)$ on tumor cells and tumor neovascularization.

First, DPSE-PEG $2 \mathrm{k}-\mathrm{iRGD}$ was chemically synthesized and characterized before the preparation of iRGD-modified RM-derived vehicles (Figure S1). DPSE-PEG ${ }_{2 \mathrm{k}}$-iRGD was prepared by combining DPSE-PEG $\mathrm{P}_{2 \mathrm{k}}$-NHS and $\mathrm{iRGD}$. The ${ }^{1} \mathrm{H}-\mathrm{NMR}$ assay showed that there were characteristic peaks of DSPE, PEG, and iRGD in the DPSE-PEG $2 \mathrm{k}-\mathrm{iRGD}$ spectrum (Figure S2), and the MALDI-TOP mass spectra displayed the molecular mass of DSPE-PEG2K-iRGD m/z at 3748.6 (Figure S3), suggesting that iRGD was successfully linked with DPSE-PEG $2 \mathrm{k}_{\mathrm{k}}$-NHS. Then, iRGDmodified RM (iRGD-RM) was successfully prepared by co-incubation with DPSE-PEG $2 \mathrm{k}$-iRGD and RM. To quantitatively evaluate the amount of DPSE-PEG-iRGD inserted into the RM, FITC was conjugated to DPSE$\mathrm{PEG}_{2 \mathrm{k}}$-NHS. The insertion efficiency of $15 \mu \mathrm{g}$ DPSEPEG-FITC into the RM from $200 \mu \mathrm{L}$ of whole blood was approximately 55\% (Figure S4). As shown in Figure 3A, the average diameter of the iRGD-RM particles was $\sim 3800$ $\pm 350 \mathrm{~nm}$. After sonication for $5 \mathrm{~min}$, the iRGD-RM diameters decreased to $\sim 1500 \pm 200 \mathrm{~nm}$ (Figure 3A), continuous extrusion with $400 \mathrm{~nm}$ and $200 \mathrm{~nm}$ filters resulted in iRGD-RM particles with average diameters of $\sim 400 \pm$ $20 \mathrm{~nm}$ and $\sim 200 \pm 10 \mathrm{~nm}$ (Figure 3A), respectively. The surface zeta potentials of RM and iRGD-RM were negative regardless of sonication or extrusion (Figure 3B). To investigate the coating ratio of nanoparticles with $\mathrm{RBC}$ membranes, the RBC membranes were added to solutions of varying MSN weights, with increasing RM volume ratios (MSN:RM) of 1:50, 1:100, 1:150, 1:200, 1:250 and 1:300; 


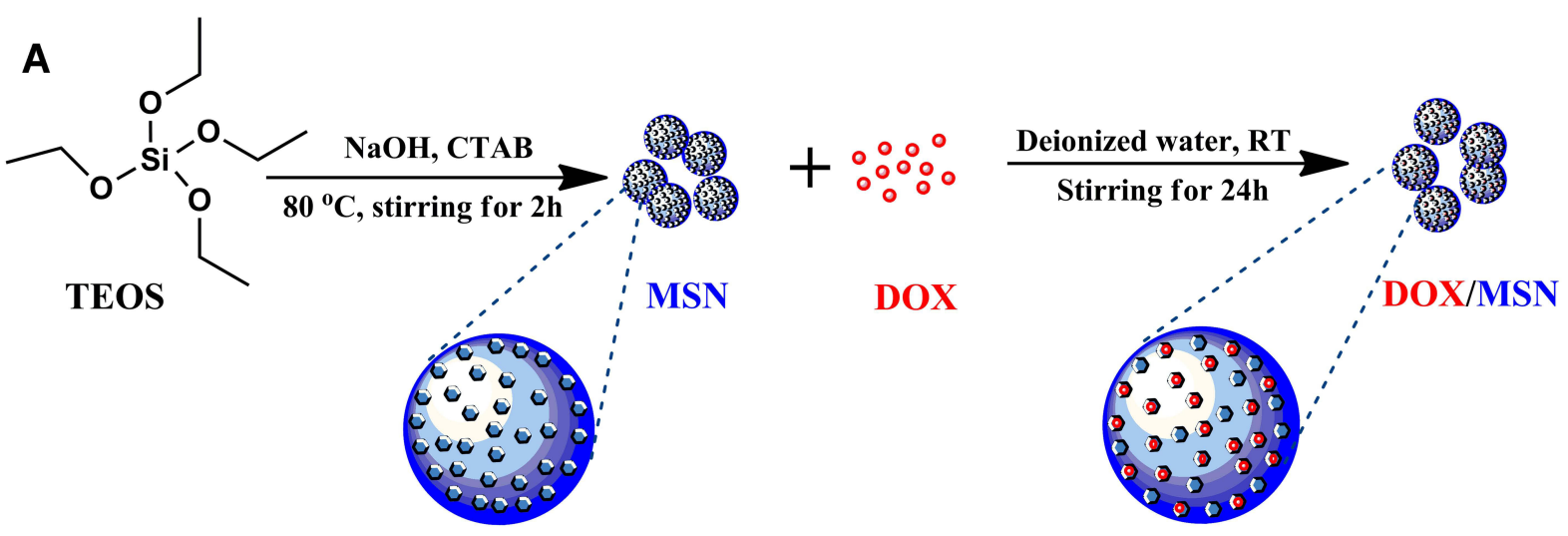

B

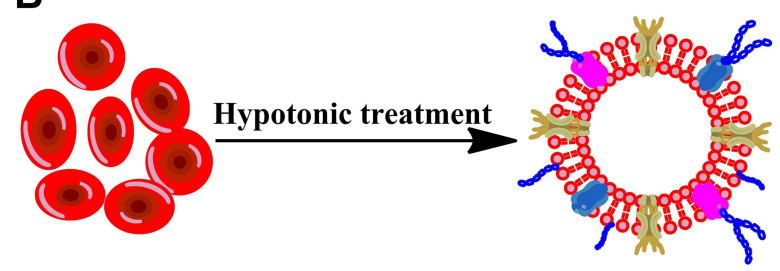

Red blood cells

RBC membrane

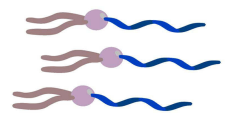

(1) Sonication: $50 \mathrm{~Hz}, 100 \mathrm{~W}, 5 \mathrm{~min}$

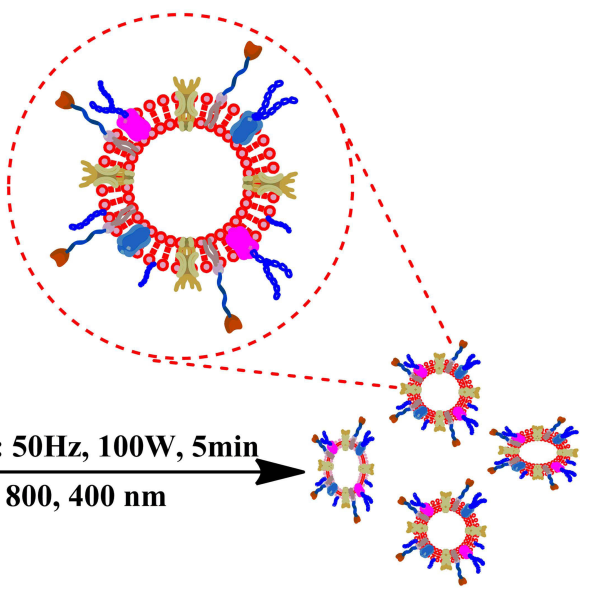

DSPE-PEG
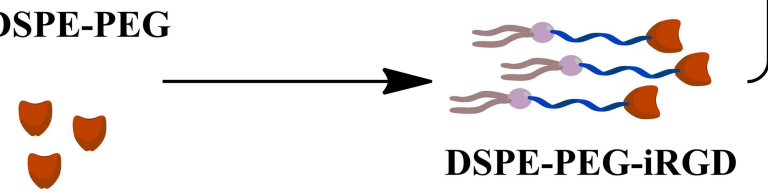

(2) Extrusion: 800, $400 \mathrm{~nm}$

iRGD inserted RBC

DSPE-PEG-iRGD

iRGD

C

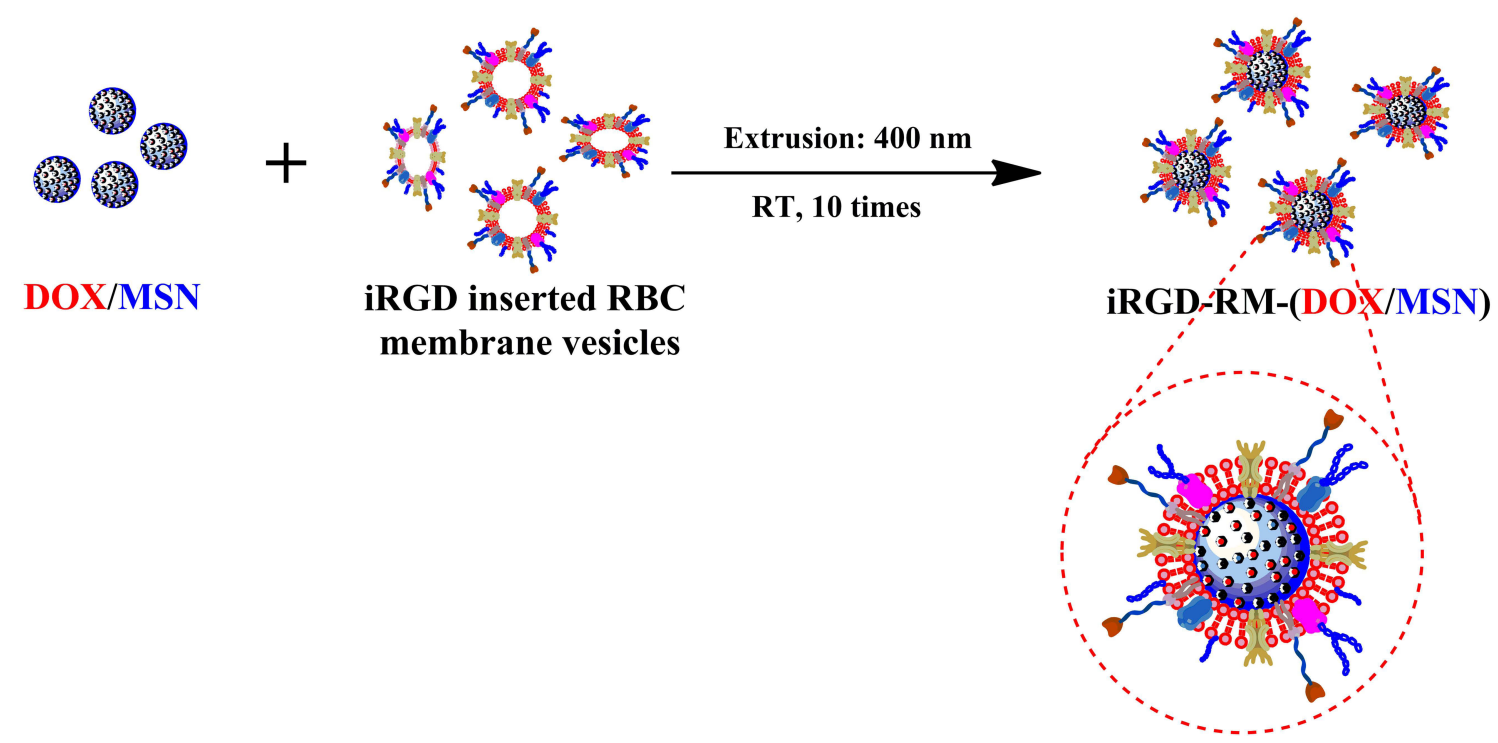

Figure I Schematic illustration of the fabrication process of iRGD-modified RBC membrane-coated mesoporous silica nanoparticles. (A) Synthesis of mesoporous silica nanoparticles (MSNs) and preparation of doxorubicin-loaded nanoparticles (DOX/MSNs); (B) Preparation of iRGD-inserted RBC membrane vesicles; (C) The fusion of DOX/ MSNs and iRGD-RBC vesicles to prepare iRGD-RM-(DOX/MSNs). 

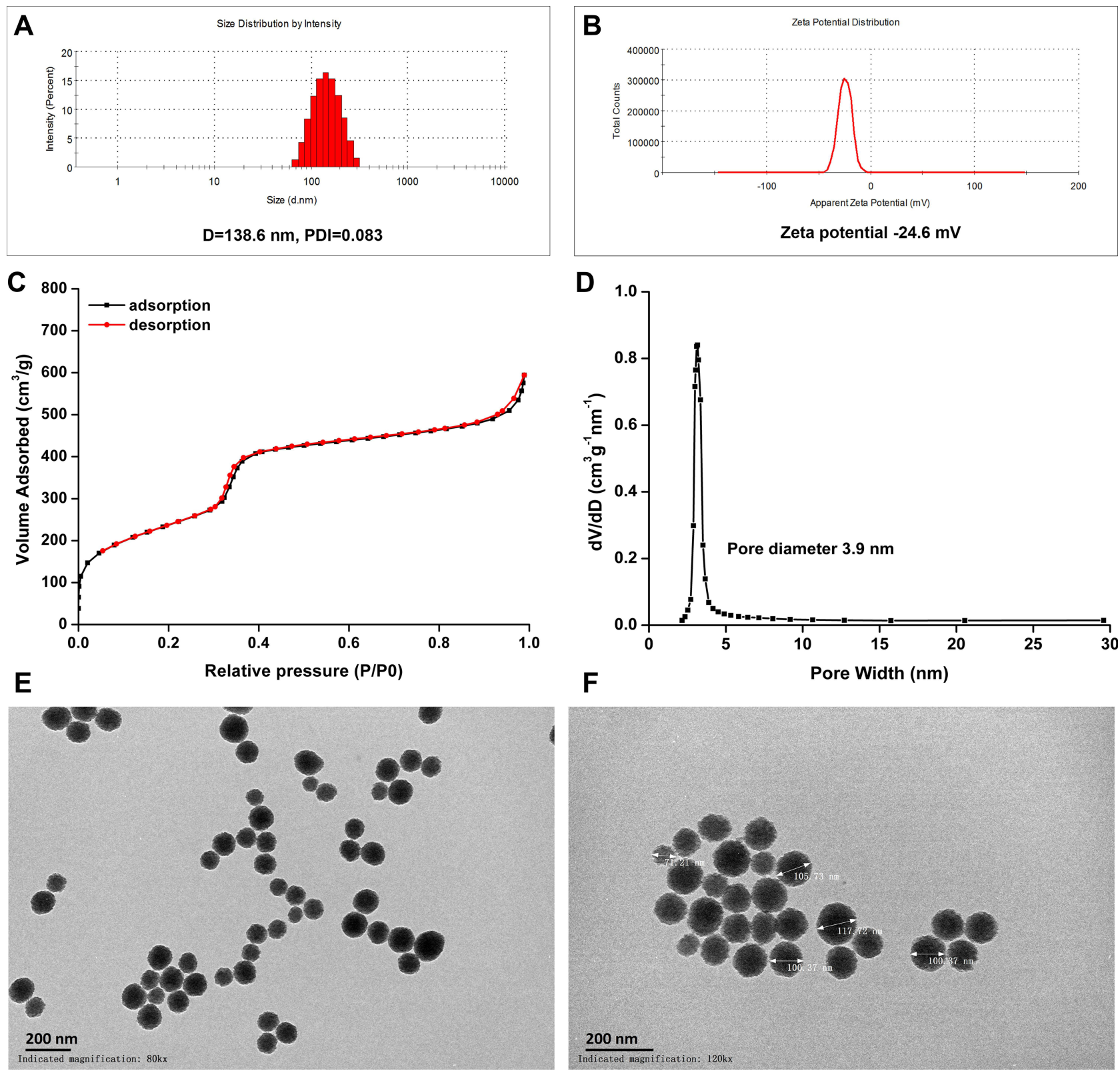

Figure 2 Characterization of mesoporous silica nanoparticles. (A) Size distribution and (B) zeta potential of MSNs; (C) $\mathrm{N}_{2}$ adsorption/desorption isotherm curves and (D) pore diameter; TEM image of MSNs, (E) and $(\mathbf{F})$ at magnifications of $80 \mathrm{k} \times$ and $120 \mathrm{k} \times$, respectively. Bar $=200 \mathrm{~nm}$.

and the mixtures were subjected to continuous extrusion to obtain RBC membrane-coated MSNs (RM-MSNs). As shown in Figure 3C and D, the size of RM-MSNs (MSN: $\mathrm{RM}=1: 200)$ was $\sim 195 \pm 10 \mathrm{~nm}$ and the zeta potential of RM-MSNs (MSN:RM=1:200) was $\sim-25 \pm 2 \mathrm{mV}$. TEM images of RM-MSNs (Figure 3E) and iRGD-RM-MSNs (Figure 3F) negatively stained with 5\% phosphotungstic acid showed a characteristic core-shell structure. The average thickness of the outer shell RBC membrane approximately $10 \mathrm{~nm}$, which is consistent with the reported thickness of the RBC membrane (approximately 5 10 $\mathrm{nm})$. By utilizing SDS-PAGE, we tested the composition of proteins on the surface of the nanoparticles. As shown in Figure S5, RM-(DOX/MSN), iRGD-RM and iRGD-RM -(DOX/MSN) exhibited protein bands similar to those found in RM, indicating the effective translocation of red blood cell membranes on the surface of iRGD-RM-(DOX/ MSN) nanoparticles. These results demonstrated the successful coating of MSN with RBC membranes or iRGDmodified RBC membranes. 

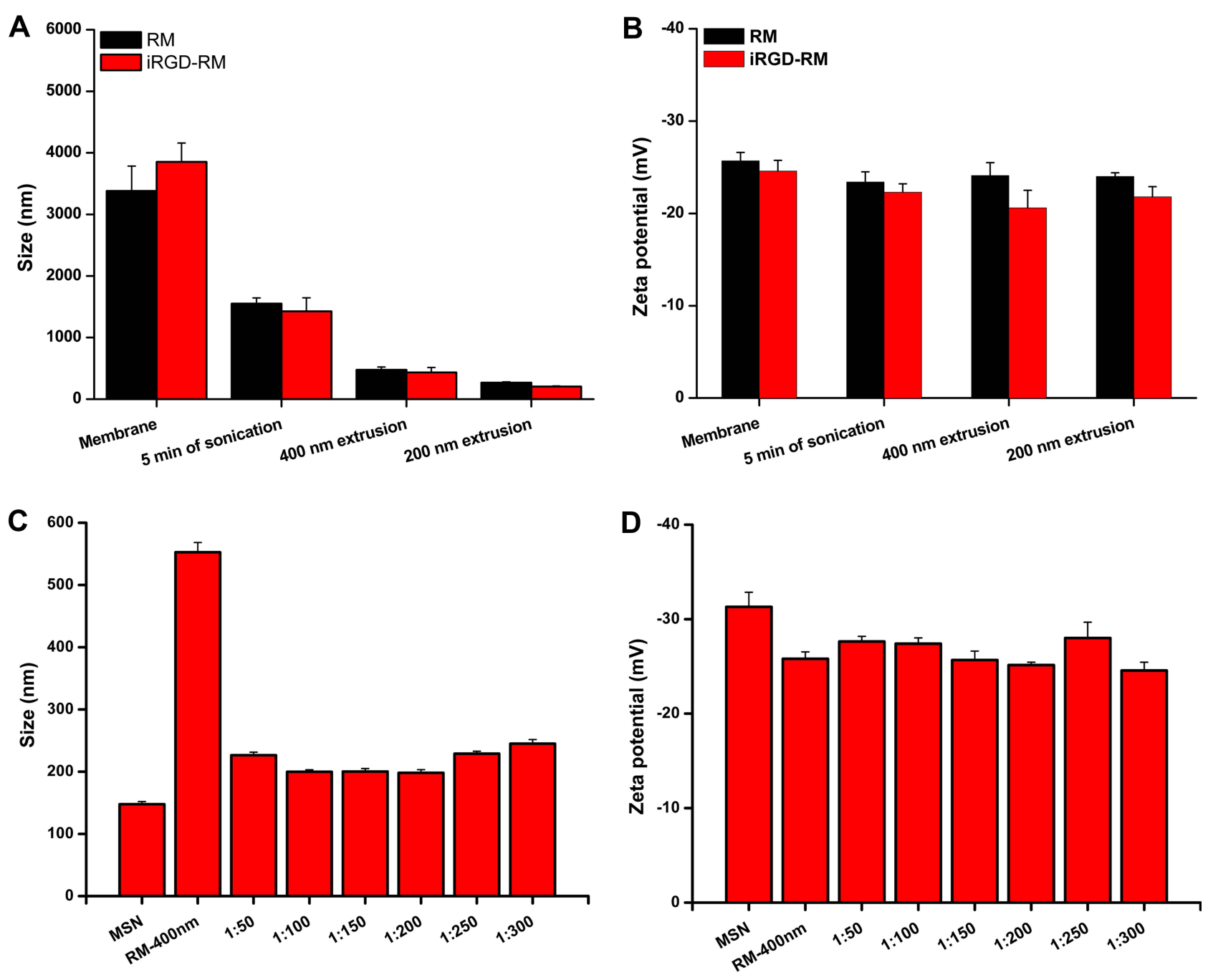

$\mathbf{E}$

MSN/RBC membrane (mg MSN/ $\mu$ l blood)
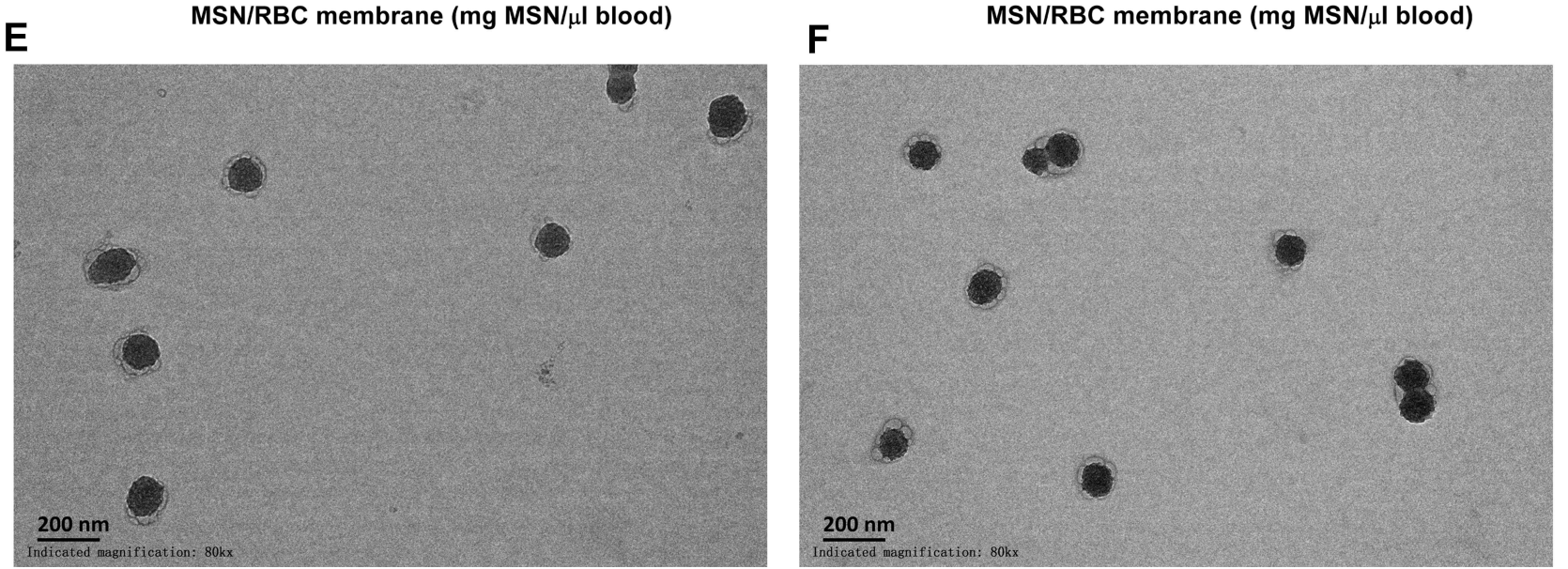

Figure 3 Preparation and characterization of RBC membrane coated mesoporous silica nanoparticles. (A) Z-average diameter and (B) zeta potential of RBC membrane vesicles or iRGD-modified RBC membrane vesicles $(n=3)$; (C) Z-average diameter and (D) zeta potential of membrane-coated nanoparticles fabricated with varying rations of MSN weight to blood volume $(n=3)$; TEM image of $(E)$ RBC membrane and $(\mathbf{F})$ iRGD-inserted RBC membrane-coated nanoparticles at a magnification of $80 \mathrm{k} \times$, Bar $=$ $200 \mathrm{~nm}$. 


\section{Characterization and Drug Release of RBC Membrane-Coated DOX-Loaded MSNs}

The diameter of drug-loaded MSNs was not affected after DOX loading, but it increased by $25-40 \mathrm{~nm}$ after RBC membrane coating (Figure 4A). DOX loading also did not affect by the zeta potential of the MSNs (Figure 4B). The textural properties of DOX/MSNs, RM-(DOX/MSNs) and iRGD-RM-(DOX/MSNs) were verified by nitrogen adsorption-desorption isotherms and pore size distribution curves. After drug loading and encapsulation of the MSNs, the pores of the MSNs disappeared (Figure S6), which proved that DOX was successfully encapsulated in MSNs and that the RBC membrane and iRGD-modified RBC membrane were successfully coated on the surface of MSNs. The particle states showed good nanometer characteristics and good dispersion with regard to drug loading and coating the RM (Figure 4C). The drug loading
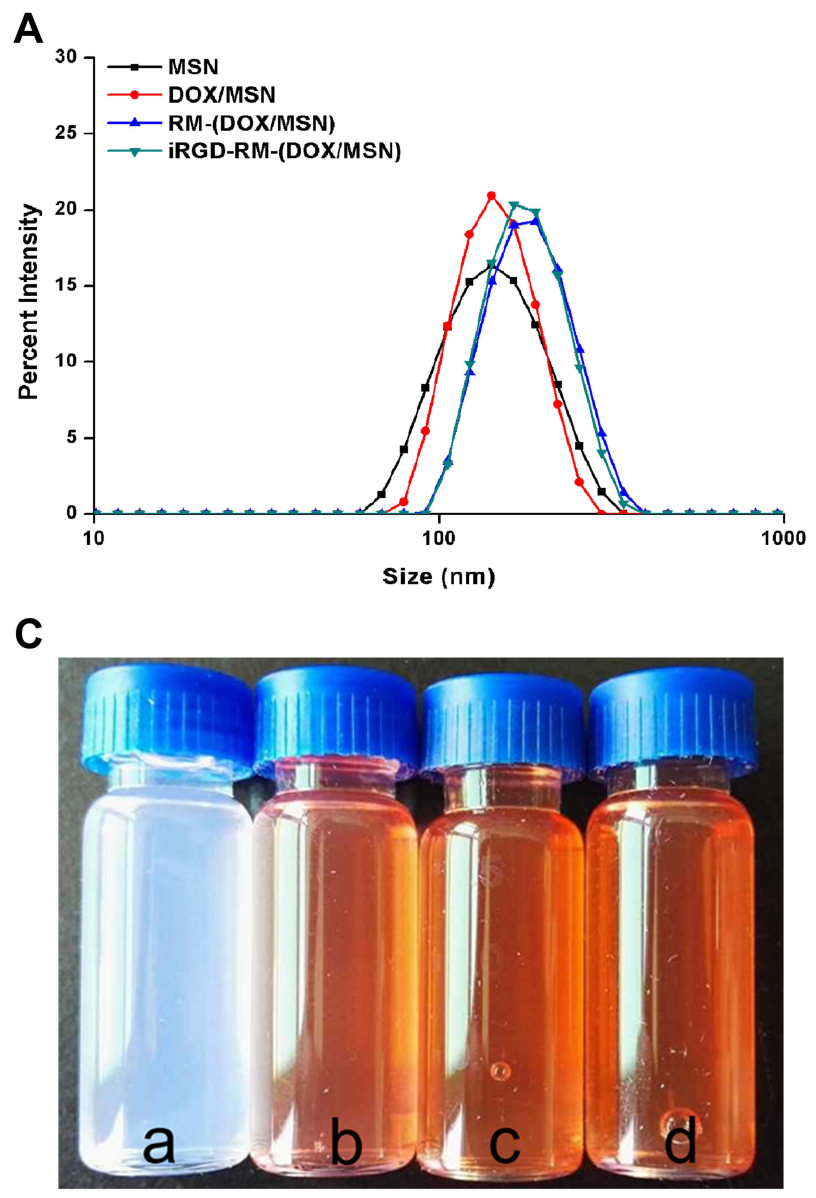

efficiency of DOX was $8.37 \%$, and the drug encapsulation efficiency was $99.55 \%$.

The drug release profiles of DOX-loaded nanoparticles showed a biphasic release pattern, which involved an initial burst release followed by sustained release (Figure 4D). The release profiles were distinctive among the different formulations. Compared with the DOX/MSN group, the RM-coated groups (RM-(DOX/ MSN) and iRGD-RM-(DOX/MSN)) showed a relatively slow drug release pattern. In addition, DOX release from all the formulations was significantly faster at $\mathrm{pH} 5.0$ than at $\mathrm{pH}$ 7.4. Thus, the drug release could slower while in the circulation and faster once the particles reach the tumor site. These findings indicated that iRGDRM-(DOX/MSNs) were released faster and more quickly in the pH 5.0 environment, which is a benefit for cancer therapy.
B

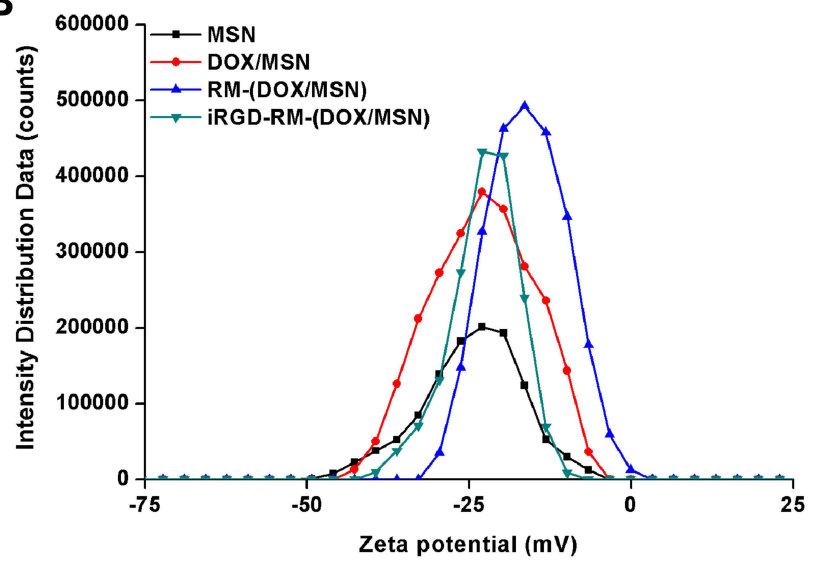

D

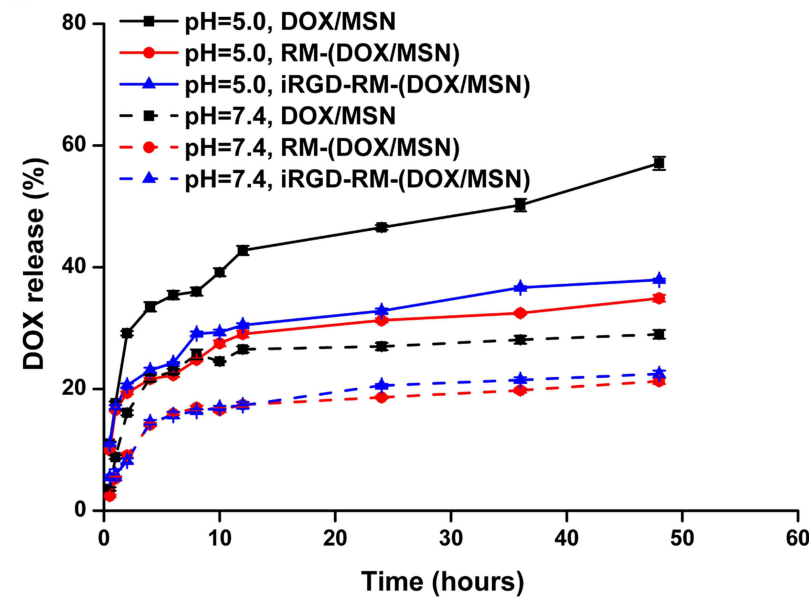

Figure 4 Preparation and characterization of RBC membrane-coated DOX-loaded mesoporous silica nanoparticles. (A) Size distribution and (B) zeta potential of the different nano-formulations; (C) color of different formulations distributed in water (a. MSNs; b. DOX/MSNs; c. RM-(DOX/MSNs); d. iRGD-RM-(DOX/MSNs)); (D) In vitro DOX release profile of different drug-loaded nanoparticles in PBS $(\mathrm{pH} 5.0$ or 7.4$)$ at $37^{\circ} \mathrm{C}(\mathrm{n}=3)$. 


\section{iRGD-RM-MSNs Have Excellent Blood Compatibility}

Evaluating the hemocompatibility of nanoparticles is the key to the design of nanoparticles for in vivo drug applications. Erythrocyte composure can be used to evaluate hemocompatibility. Erythrocytes are used as an ideal reference standard for osmotic pressure changes. When the blood is disturbed by osmotic pressure or physical changes, the erythrocytes are destroyed. The destruction of erythrocytes leads to a series of reactions induced by the release of hemoglobin, which is an indicator of blood poisoning. Therefore, the swelling and rupture of erythrocytes is a good parameter to evaluate the toxicity of foreign substances to blood cells. Blood compatibility is necessary for drug delivery throughout the body when they are administrated intravenously. ${ }^{43}$ In this study, each group of nanoparticles was incubated in diluted mouse blood for $3 \mathrm{~h}$ at $37{ }^{\circ} \mathrm{C}$ (Figure 5C). As shown in Figure $5 \mathrm{~A}$ and $\mathrm{B}$, more than $80 \%$ hemolysis was induced in the MSN group when concentration of MSNs was $250 \mu \mathrm{g} / \mathrm{mL}$. When the concentration of MSNs reached 250 $\mu \mathrm{g} / \mathrm{mL}$ in the RM-MSN and iRGD-RM-MSN group, the hemolysis rates were negative, which suggests significant improvement in the hemolysis performance compared to that of independent MSN nanoparticles. Additionally, the
A
Lo
$\Sigma$

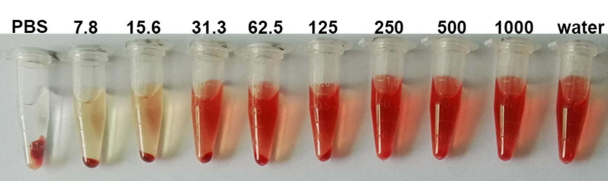

$\sum$
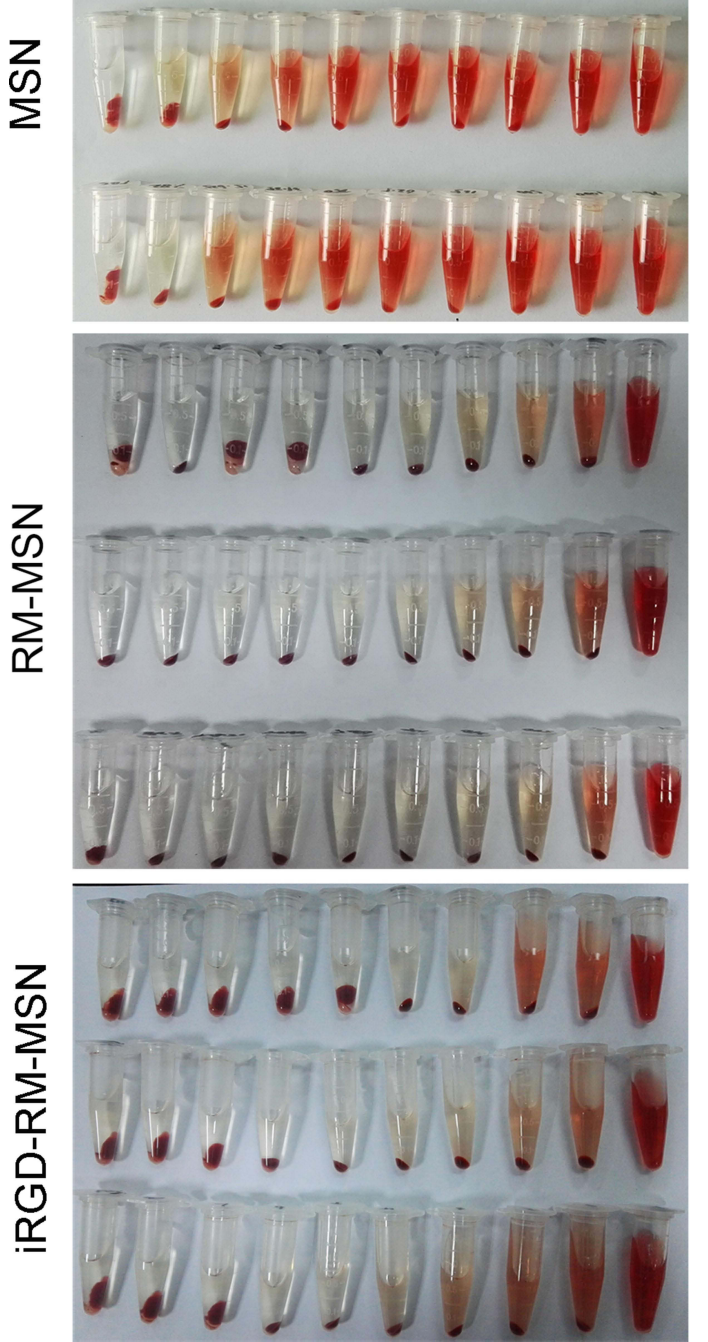

$B_{120}$

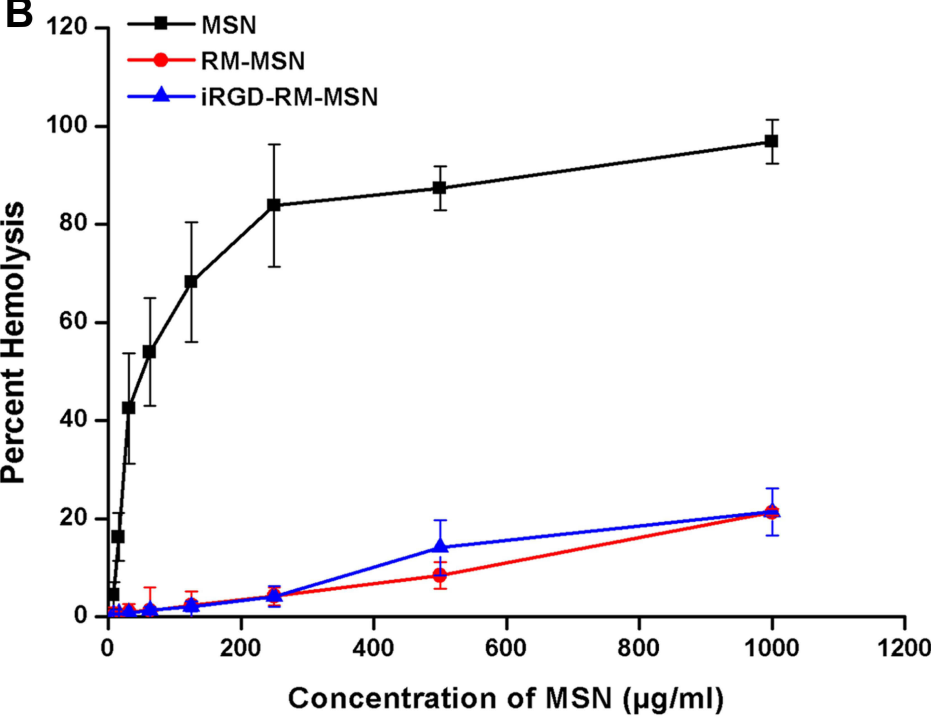

C

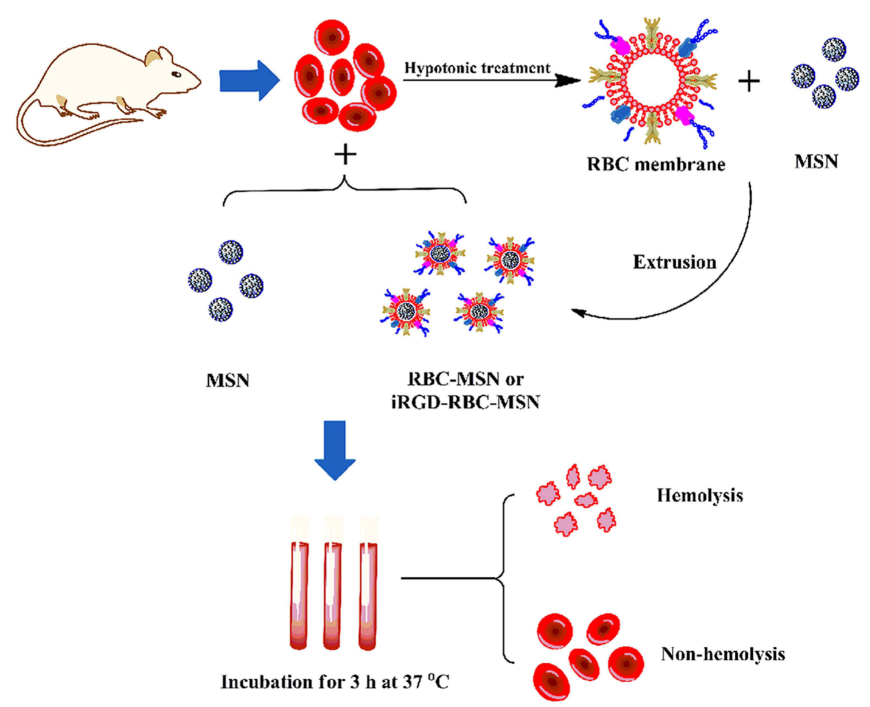

Figure 5 Hemolysis assay of MSNs, RM-MSNs and iRGD-RM-MSNs (A) Color of hemolysis at concentrations ranging from 7.8 to $1000 \mu g / m L$. Samples were treated with water or Ix PBS as a positive and negative control, respectively. (B) Quantitative characterization of hemolysis $(n=3)$; $(\mathbf{C})$ Schematic illustration of the hemolysis assay. 
adsorption of plasma protein by RM-MSNs and iRGD-RMMSNs was greatly reduced compared with that of MSNs (Figure S7). The RM-coated nanoparticles (RM-MSNs and iRGD-RM-MSNs) have excellent blood compatibility and satisfy the conditions for safe intravenous injection.

\section{iRGD-RM-(DOX/MSNs) Have No Effects on Inflammatory Cytokine Secretion and Acute Toxicity}

To further evaluate the acute toxicity and immunogenicity of nanoparticles, mice were systemically administrated a high dose of the nano-formulations $(30 \mathrm{mg} / \mathrm{kg}$ DOX, three times higher than the common dose). The results of the blood analysis showed that the formulations had almost no influence on MPV, MCV, HGB or the number of RBCs
(Figure 6A, D-F). Compared with the control group, the DOX/MSN showed significant decreases in the number of WBCs and PLTs at 12 or 24 hours after administration, whereas this decline was negligible in the RM-(DOX/ MSN) and iRGD-RM-(DOX/MSN) treated groups (P > 0.05) (Figure 6B and C). These results reveal that injection of RM-(DOX/MSNs) or iRGD-RM-(DOX/MSNs) induces a lower inflammatory response than do DOX/MSNs.

Additional information was obtained by measuring the serum level of cytokines using ELISA kits. As shown in Figure 6G-I, injection of DOX/MSNs stimulated higher levels of inflammation-related cytokines after $12 \mathrm{~h}$ and 24 $\mathrm{h}$, including interleukin-1 $\beta$ (IL-1 $\beta$ ), interleukin-6 (IL-6), and tumor necrosis factor- $\alpha(\mathrm{TNF}-\alpha)$, than those after injection of RM-(DOX/MSNs) or iRGD-RM-(DOX/MSNs). However, there was no significant difference in the levels
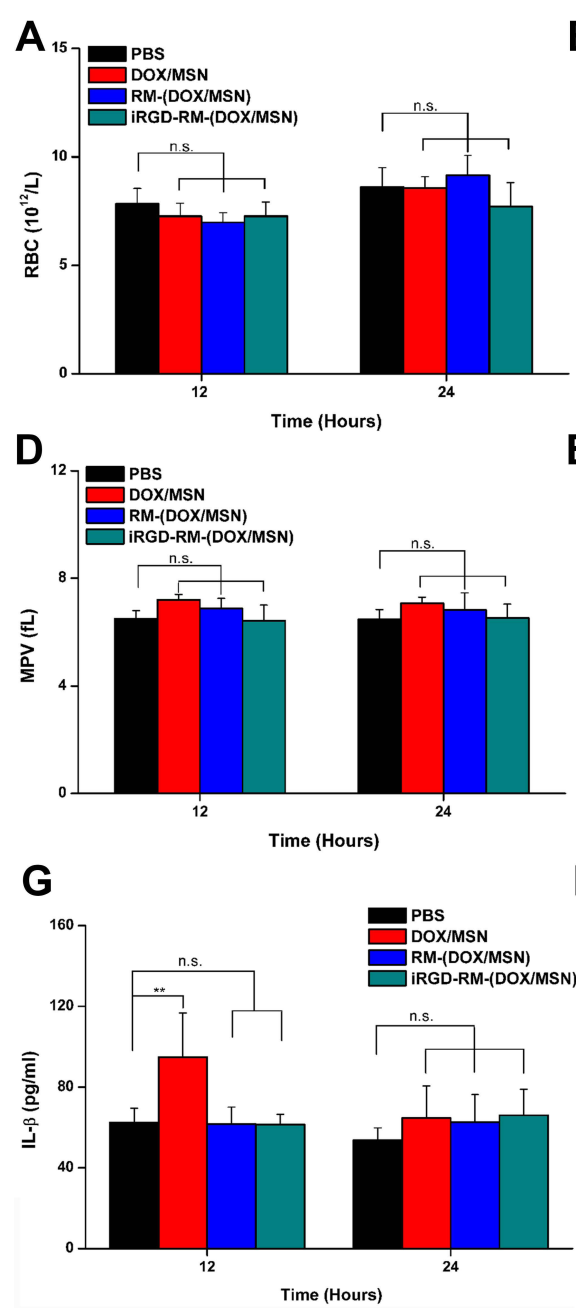
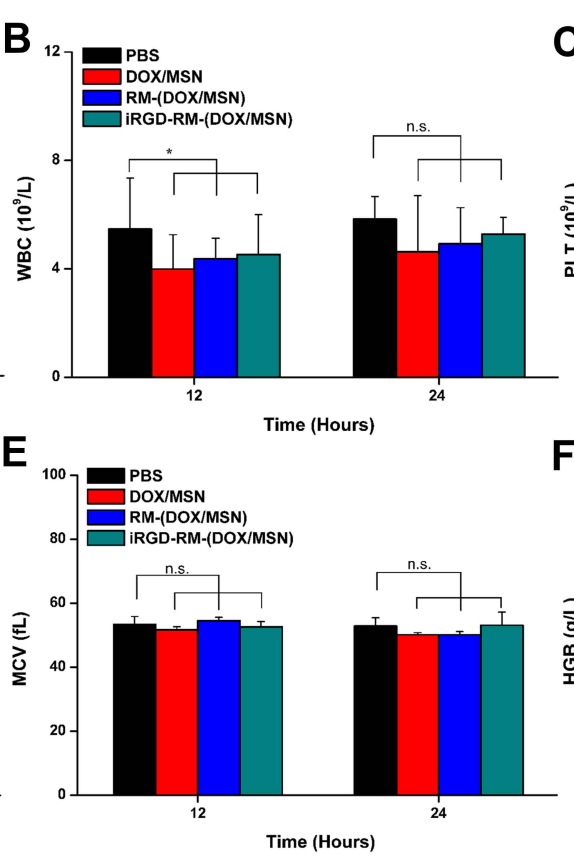

H

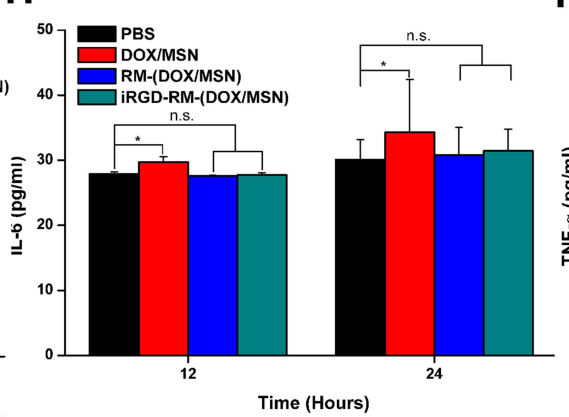

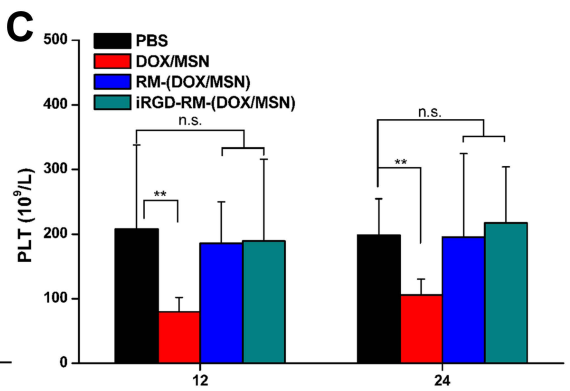

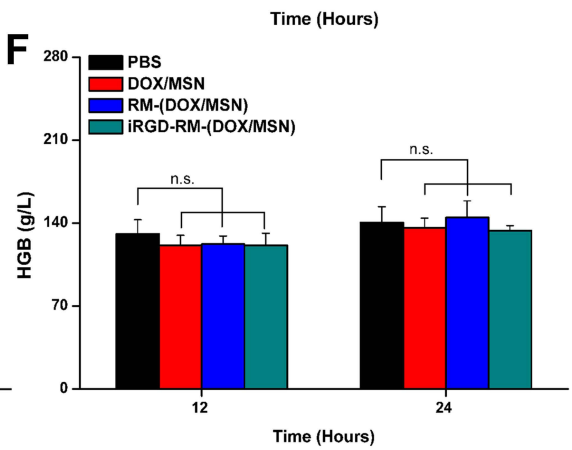

I

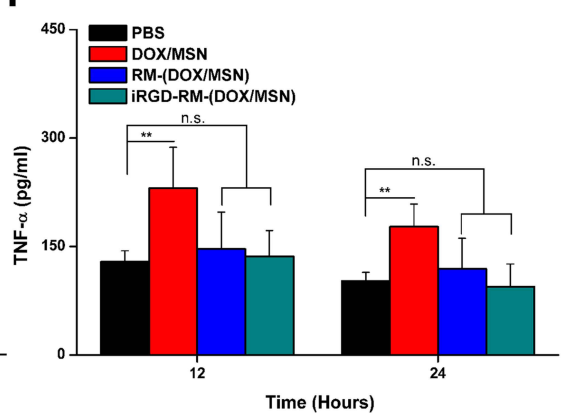

Figure 6 In vivo and acute toxicity and immunogenicity. Mice were intravenously administered a high dosage of different formulations (30 mg/kg DOX), and blood samples were collected at 12 and 24 hours after administration. (A) RBC, (B) WBC, (C) PLT, (D) MPV, (E) MCV, (F) HGB levels and (G-I) serum levels of the main proinflammatory cytokines (IL-I $\beta$, IL-6, and TNF- $\alpha$ ). Bars are represented as SD, ${ }^{n . s} p>0.05$, ${ }^{*} p<0.05$, **p $<0.0$ I $(n=3)$. 
of inflammatory cytokines in the RBC membrane-coated groups compared with the control group. Such results suggest that coating nanoparticles with $\mathrm{RBC}$ membranes is a potential approach to improve the blood compatibility of MSNs and reduce the inflammatory response.

\section{Low Cellular Uptake of iRGD-RM-(DOX/ MSNs) on RAW264.7 Cells}

To further investigate the escape of RBC membrane-coated DOX/MSNs from phagocytosis by immune cells, the cellular uptake of different formulations in RAW264.7 mouse mononuclear macrophage cells was examined by fluorescence microscopy and flow cytometry. As shown in Figure 7A, only weak red fluorescence of DOX could be observed for the DOX-free group. However, the fluorescence intensity of the DOX/MSN group was the strongest, which might be because the nanoparticles are more easily taken up by cells than free drugs and the MSNs contain a large amount of DOX. Compared with the DOX/MSN group, the RBCcoated nanoparticle groups (RM-(DOX/MSN) and iRGDRM-(DOX/MSN)) had significantly reduced uptake behavior, indicating that the RBC-coated formulations achieve the function of escaping phagocytosis by immune cells. The FCM results were consistent with those of fluorescence
A
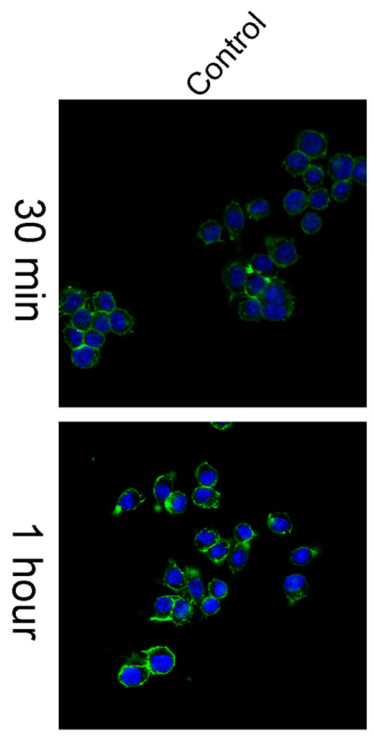

B

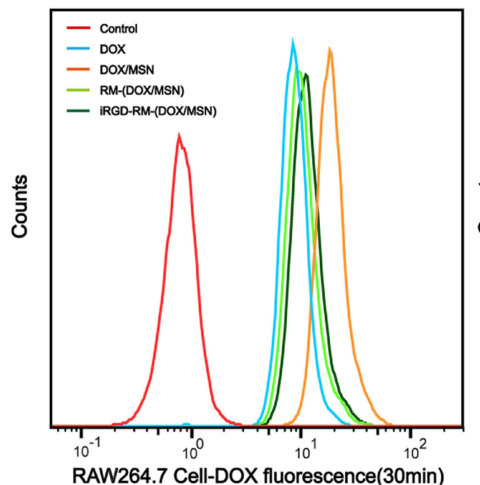

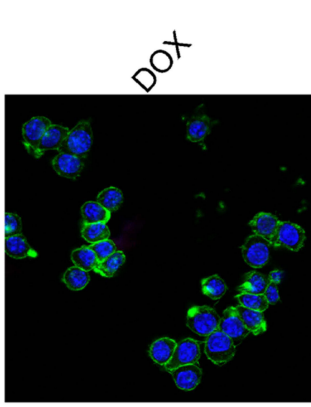

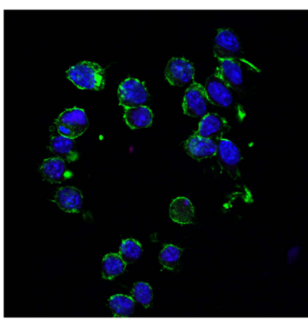

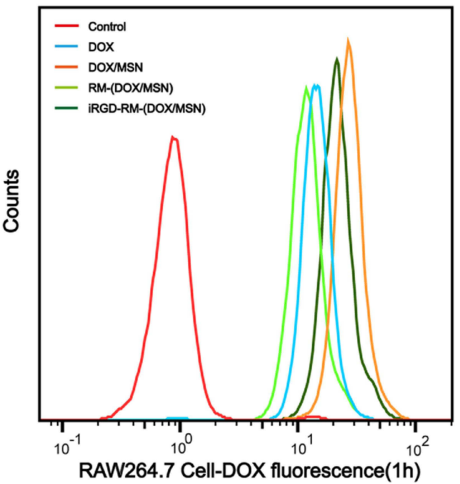

C

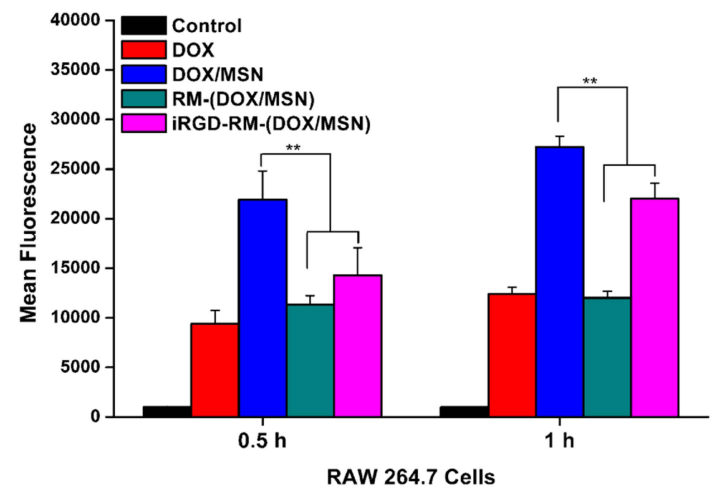

Figure 7 In vitro cellular uptake of DOX-loaded nanoparticles by RAW264.7 cells at 30 min and I hour after treatment. (A) Images were observed by CLSM. The nucleus and F-actin were stained with DAPI (blue) and phalloidin (green), respectively. DOX exhibits red fluorescence. (B) Flow cytometry histograms of cells treated with different formulations. (C) Quantification of the mean fluorescence intensity of the histograms in (B). ${ }^{* *} \mathrm{p}<0.01$, the two groups were compared by independent Sample $t$-test ( $\mathrm{n}$ $=3)$. 
microscopy, which showed that the RBC coated nanoparticles had reduced uptake by macrophages (Figure 7B and C), thus achieving immune cell escape.

\section{iRGD-RM-(DOX/MSNs) Effectively Exhibit Tumor-Targeting Capability and Cytotoxicity in Breast Cancer}

It has been reported that iRGD is a tumor-penetrating peptide that shows tissue-specific penetration ability, which improves the targeting ability toward cancer cells. $^{44,45}$ It specifically binds to $\alpha v \beta 3$ or $\alpha v \beta 5$ integrin on tumor vascular endothelial cells. ${ }^{13}$ To evaluate the targeting uptake ability of iRGD-RM-(DOX/MSNs), we used fluorescence microscopy and FCM. As shown in Figure $8 \mathrm{~A}$, the red fluorescence intensity of DOX in
MDA-MB-231 cells was higher in the iRGD-RM-(DOX/ MSN) group, indicating that the iRGD peptide possesses a high affinity for cancer cells. To further validate the effect of the targeting peptide on the endocytosis of the nanoparticles, competitive treatments with a high dose of iRGD were performed. The fluorescence intensity was significantly reduced in the iRGD-RM-(DOX/MSN) + iRGD group, in which MDA-MB-231 cells were treated with excessive iRGD in advance for half an hour before treatment with nanoparticles (Figure 8A). Meanwhile, the cellular uptake ability of NPs in MDA-MB-231 cells was quantitatively analyzed by FCM. The FCM results were consistent with those of fluorescence microscopy (Figure $8 \mathrm{~B}$ and $\mathrm{C}$ ). Although the DOX/MSN group had a high mean fluorescence intensity in MDA-MB-231 cell,
A
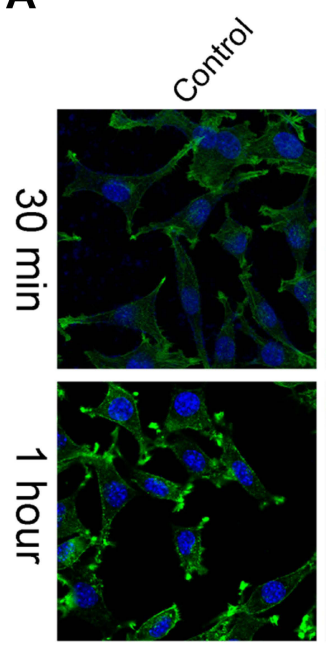

B

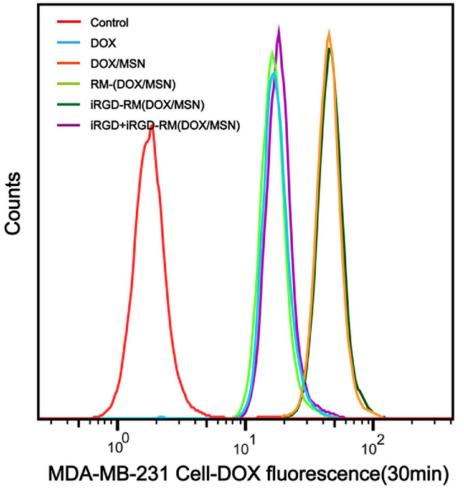

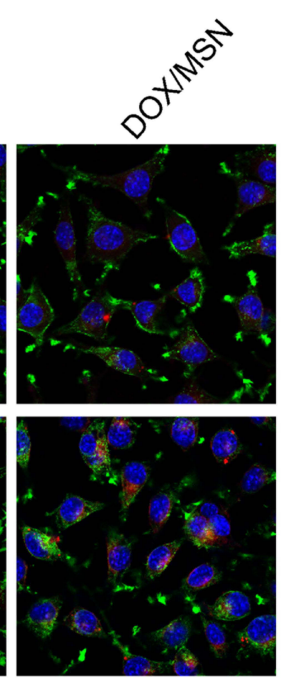
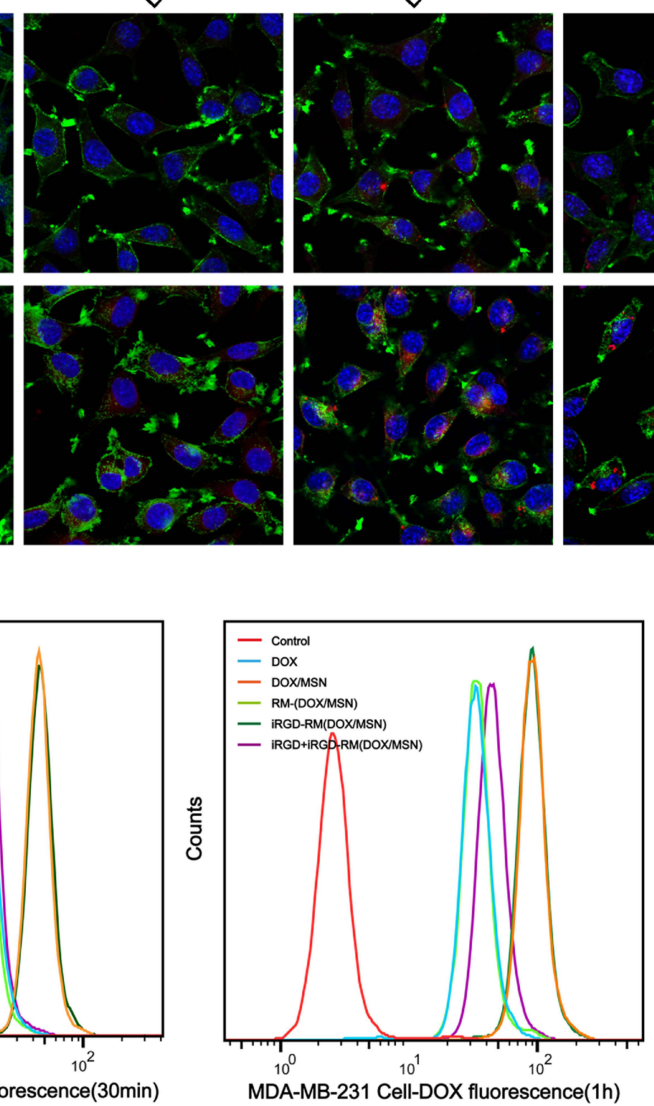

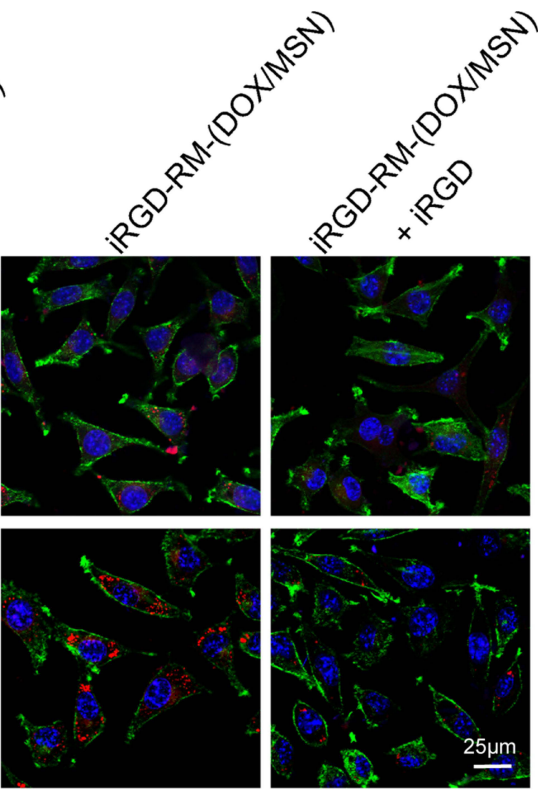

C

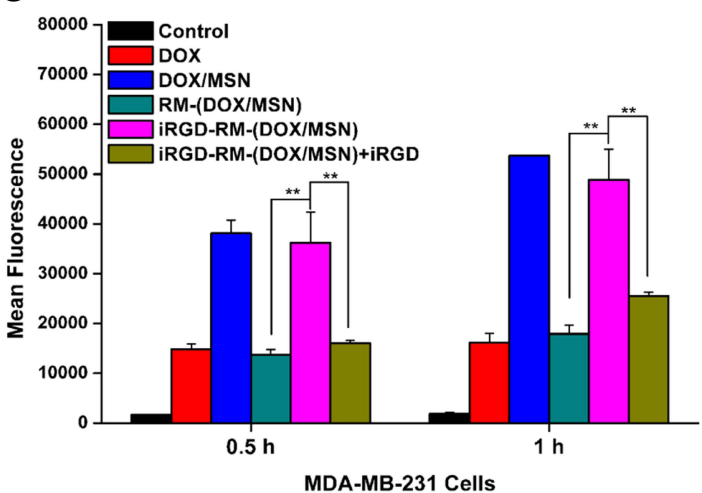

Figure 8 In vitro cellular uptake of DOX-loaded nanoparticles by MDA-MB-23I cells at 30 min and I hour after treatment. (A) Images were observed by CLSM. The nucleus and F-actin were stained with DAPI (blue) and phalloidin (green), respectively. DOX exhibits red fluorescence. (B) Flow cytometry histograms of cells treated with different formulations. (C) Quantification of the mean fluorescence intensity of the histograms in (B). **p $<0.0$, the two groups were compared by independent Sample $t$-test $(n=3)$. 
it could not avoid immune escape as indicated by the high uptake in immune cells (Figure $7 \mathrm{~A}-\mathrm{C}$ ). However, the iRGD-RM-(DOX/MSN) group not only showed enhanced uptake into cancer cells but also avoided uptake into immune cells to achieve efficient targeting.

To further visualize the tumor-targeted iRGD-RMMSNs, DiD was loaded inside MSNs, and the fluorescence of DiD was monitored using an in vivo imaging system. In brief, DiD-labeled MSNs and iRGD-RM-MSNs were intravenously injected into mice with in situ breast cancer. DiD signals were detected at different time points in live mice and excised tumors. As shown in Figure S8A, the fluorescence signal of DiD/MSNs was observed at 2-4 h, and the fluorescence signal of iRGD-RM-(DiD/MSNs) was observed at 8-24 h. Compared with the DiD/MSN group, the iRGD-RM-(DiD/MSN) group displayed a stronger fluorescence intensity in the tumor (Figure S8B), indicating an excellent targeting ability of iRGDmodified RM-coated nanoparticles.

Then, the apoptosis-inducing effect of different formulations on MDA-MB-231 cells was also investigated. First, the toxicity of the different formulations on MDA-MB -231 cells was detected by MTT (Figure 9A). Cell viabilities were found to be greater than $85 \%$ when the concentration of MSNs was less than $100 \mu \mathrm{g} / \mathrm{mL}$, which demonstrated the low toxicity of the nanoparticles. However, the DOX-loaded nanoparticles exhibited clear toxicity toward MDA-MB-231 cells in a dose-dependent manner and were more potent than free DOX (Figure 9B). In addition, compared with other groups, the iRGD-RM -(DOX/MSN) group showed enhanced antitumor effects. When excess iRGD was added to MDA-MB-231 cells for half an hour prior to nanoparticle treatment, the cell viability was increased (Figure 9B). The results indicated that the enhanced cytotoxicity may be due to the targeting ability of the coated nanoparticles to MDA-MB-231 cells owing to their increased intracellular concentrations of DOX.

Furthermore, an Annexin V-APC/7-AAD apoptosis detection kit and flow cytometry were used to further confirm MDA-MB-231 cell apoptosis after various treatments (Figure 9C and D). As expected, the percentage of apoptotic cells was the highest in the iRGD-RM-(DOX/ MSN) group and was significantly reduced in the group treated with excess iRGD in advance. Free DOX, DOX/ MSN, RM-(DOX/MSN), iRGD-RM-(DOX/MSN) and iRGD-RM-(DOX/MSN) + iRGD induced apoptosis rates of $12.7 \%, 27.7 \%, 16.8 \%, 71.1 \%$, and $23.3 \%$, respectively
(Figure 9D). In summary, the apoptotic and necrotic rates of MDA-MB-231 cells treated with iRGD-RM-(DOX/ MSNs) were much higher than those of the other nanoparticle formulations $(\mathrm{P}<0.05)$. These results indicated that iRGD-RM-(DOX/MSNs) possessed excellent targeting and therapeutic effects on MDA-MB-231 cells in vitro.

\section{iRGD-RM-(DOX/MSNs) Effectively Inhibit Tumor Growth Without Additional Toxic Effects}

The in vivo antitumor activity evaluation of the DOXloaded nanoparticles was implemented on the in-situ tumor model mice. Model mice were injected with various nanoparticles formulations or free DOX via tail vein on the 9 th and 12 th days postimplantation. The in vivo antitumor activity results are shown in Figure 10. Tumor growth was significantly inhibited after intravenous administration of different DOX formulations (Figure 10A). To quantitatively evaluate the antitumor effect, tumors were excised and weighed at the end of the treatment (Figure 10B). All the nanoparticle formulations exhibited more effective antitumor activity than the DOX-free group (Figure 10C and D). The tumor growth inhibition rate of the iRGD-RM-(DOX/MSN) group reached to $86.29 \pm 5$. 12\% (Figure 10D), which showed the highest inhibitory effect among the experimental groups (Figure 10C and D). In particular, the iRGD-RM -(DOX/MSN) group exhibited more enhanced antitumor efficacy than the RM-(DOX/MSN) group $(\mathrm{P}<0.01)$, which is likely attributed to the targeting function of iRGD. However, there was no significant difference between the DOX/MSN group and the RM-(DOX/MSN) group $(\mathrm{P}>0.05)$. Therefore, the iRGD-modified erythrocyte membrane biomimetic nano-administration strategy can effectively inhibit tumor growth and has good tumortargeting and antitumor activity.

The safety profiles of the DOX formulations were evaluated by measuring the changes in body weight as a function of time (Figure 10E). Body weight curves showed some decrease in DOX-treated groups compared with the control group. For statistical analysis, the body weights before injection and at the end of the experiment were compared (Figure 10F). There was an appreciable loss in the body weight of tumor-bearing mice after treatment with free $\mathrm{DOX}(\mathrm{P}<0.05)$ or $\mathrm{DOX} /$ MSNs $(\mathrm{P}<0.01)$, whereas no significant difference was observed mice treated with RM-(DOX/MSNs) or 

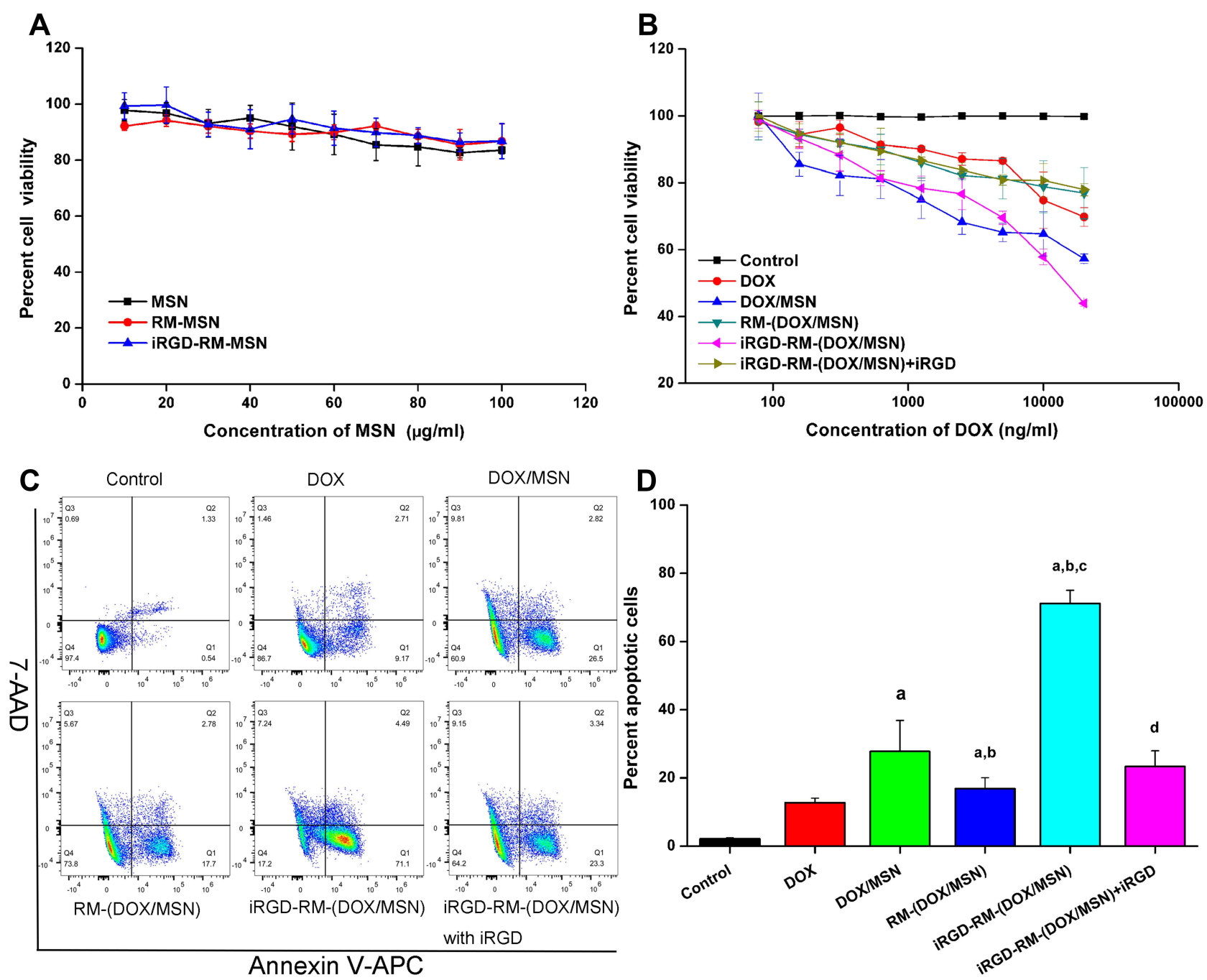

Figure 9 In vitro cytotoxicity of nanoparticles in MDA-MB-23 I cells. Cell viability of (A) empty nanoparticles and (B) DOX-loaded nanoparticles in MDA-MB-23 I cells at 48 h. Bars represent the SD $(n=6)$. (C) Representative flow cytometric patterns and $(\mathbf{D})$ quantitative analysis of apoptosis by flow-cytometric analysis. MDA-MB-23I cells were treated with free DOX, DOX/MSNs, RM-(DOX/MSNs), iRGD-RM-(DOX/MSNs) and iRGD-RM-(DOX/MSNs) with free iRGD for $48 \mathrm{~h}$ at a DOX concentration of 10 $\mu \mathrm{g} / \mathrm{mL}$. ${ }^{\mathrm{a}} \mathrm{p}<0.001$ vs DOX; ${ }^{\mathrm{b}} \mathrm{p}<0.01$ vs DOX/MSN; ${ }^{\mathrm{c}} \mathrm{p}<0.001$ vs $\mathrm{RM}-(\mathrm{DOX} / \mathrm{MSN})$; ${ }^{\mathrm{d}} \mathrm{p}<0.01$ vs iRGD-RM-(DOX/MSN) $(\mathrm{n}=3)$.

iRGD-RM-(DOX/MSNs) (P>0.05). H\&E staining of the heart, liver, spleen, lung, and kidney from mice treated with saline or various nanoparticle formulations showed no histologic abnormalities (Figure S9). All the results revealed that iRGD-RM-(DOX/MSNs) are safe nanoparticles and are expected to be a safe in vivo targeted.

\section{Conclusion}

TNBC, one of the most aggressive and heterogeneous subtypes of breast cancer, is known to be associated with high recurrence and metastasis rates because of the lack of definitive targets. Novel formulation for targeted drug delivery for TNBC treatment has become an appealing therapeutic strategy. In the current study, by utilizing the tumor-targeting and tumor-penetrating properties of $\mathrm{iRGD}$ and the biomimetic properties of RBC membranes, we have successfully fabricated an iRGD-modified RBC membrane nanoparticle drug delivery system and demonstrated that this system was capable of efficient TNBC targeting and elimination with good biocompatibility. This bio-inspired nano-system of our current study provides not only a promising formulation strategy for TNBC treatment but also a beneficial referee for target treating other heterogeneous tumors. 

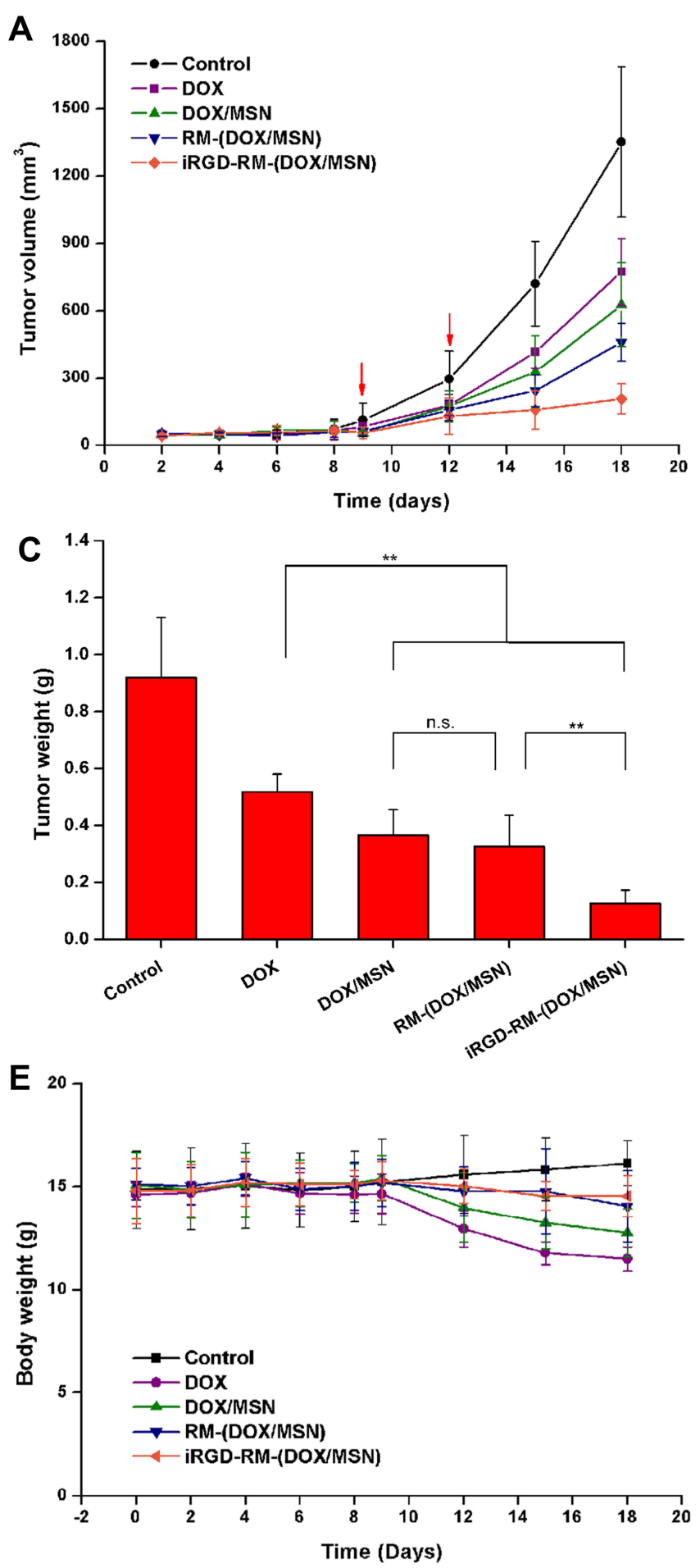

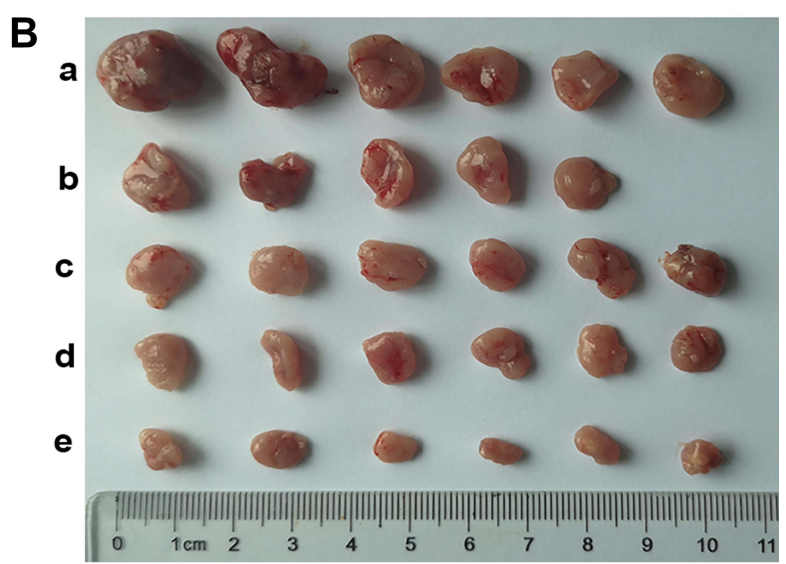

D

\begin{tabular}{ll}
\hline Groups & Tumor inhibitory rate (\%) \\
\hline DOX & $43.70 \pm 6.88$ \\
DOX/MSN & $60.22 \pm 9.80$ \\
RM-(DOX/MSN) & $64.47 \pm 11.94$ \\
iRGD-RM-(DOX/MSN) & $86.29 \pm 5.12$ \\
\hline
\end{tabular}

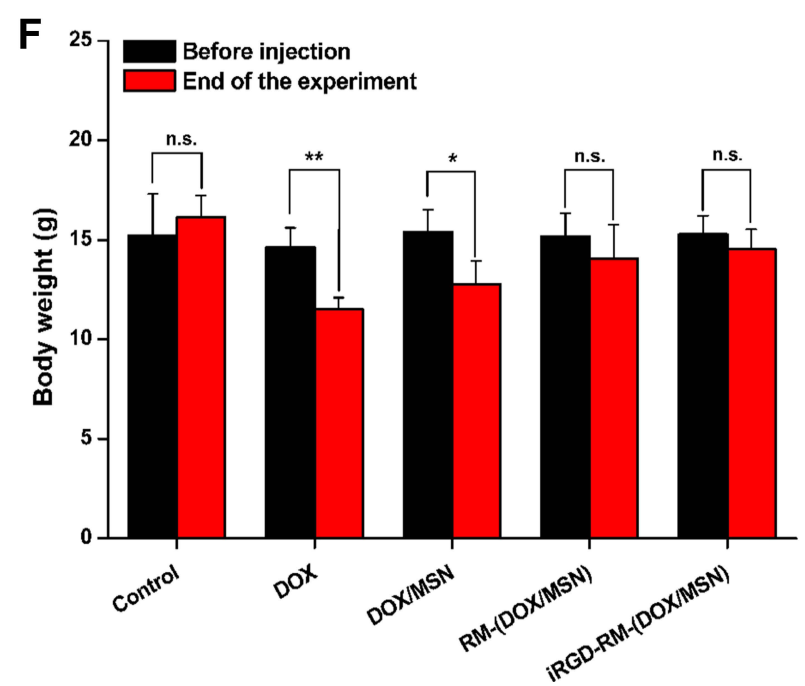

Figure 10 In vivo antitumor activity. (A) Tumor growth curves showing changes in the tumor volume. (B) Images of excised tumors (a control, b DOX, c DOX/MSN, d RM(DOX/MSN), e iRGD-RM-(DOX/MSN)) and (C) tumor weight at the end of the experiment. (D) Tumor growth inhibition rate as calculated with the excised tumor weight. (E) Alterations in total body weight of tumor-bearing mice after treatment with different nanoparticle formulations. (F) Comparison of body weight before nanoparticle treatment and at the end of the test. Bars are represented as the SD, ${ }^{n . s} p>0.05,{ }^{*} p<0.05,{ }^{* *} p<0.01 \quad(n=6)$. 


\section{Data Sharing Statement}

The authors declare that all the data supporting the findings of this study are available within the manuscript and the Supplementary data. Source data for the figures in this study are available from the authors.

\section{Acknowledgment}

We thank Rong Zhang and Zhe Zhang for their thoughtful guidance related to this study. We thank Qing Wang for language help and the other authors for their valuable opinions on article writing. We also thank the central laboratory teachers for their assistance in optimizing the instrument conditions for our experiments.

\section{Funding}

This work was supported by the National Natural Science Foundation of China (no.31600806) and the talent pool key support projects from Army Medical University (no.2019R042).

\section{Disclosure}

The authors declare no conflicts of interest.

\section{References}

1. Siegel RL, Miller KD, Fuchs HE, Jemal A. Cancer statistics, 2021. CA Cancer J Clin. 2021;71(1):7-33. doi:10.3322/caac.21654

2. Sohn J, Liu S, Parinyanitikul N, et al. cMET activation and EGFR-directed therapy resistance in triple-negative breast cancer. $J$ Cancer. 2014;5(9):745-753. doi:10.7150/jca.9696

3. Waks AG, Winer EP. Breast cancer treatment. JAMA. 2019;321 (3):316. doi:10.1001/jama.2018.20751

4. Loibl S, Poortmans P, Morrow M, Denkert C, Curigliano G. Breast cancer. Lancet. 2021;397(10286):1750-1769. doi:10.1016/S01406736(20)32381-3

5. Li N, Deng Y, Zhou L, et al. Global burden of breast cancer and attributable risk factors in 195 countries and territories, from 1990 to 2017: results from the Global Burden of Disease Study 2017. J Hematol Oncol. 2019;12(1):140. doi:10.1186/s13045-019-0828-0

6. Garrido-Castro AC, Lin NU, Polyak K. Insights into molecular classifications of triple-negative breast cancer: improving patient selection for Treatment. Cancer Discov. 2019;9(2):176-198. doi:10.1158/2159-8290.CD-18-1177

7. Pusztai L, Foldi J, Dhawan A, DiGiovanna MP, Mamounas EP. Changing frameworks in treatment sequencing of triple-negative and HER2-positive, early-stage breast cancers. Lancet Oncol. 2019;20(7):e390-e396. doi:10.1016/S1470-2045(19)30158-5

8. Borri F, Granaglia A. Pathology of triple negative breast cancer. Semin Cancer Biol. 2020;72:136-145.

9. Hwang SY, Park S, Kwon Y. Recent therapeutic trends and promising targets in triple negative breast cancer. Pharmacol Ther. 2019;199:30-57.

10. Franzoi MA, Romano E, Piccart M. Immunotherapy for early breast cancer: too soon, too superficial, or just right? Ann Oncol. 2021;32 (3):323-336. doi:10.1016/j.annonc.2020.11.022
11. Ruoslahti E. Tumor penetrating peptides for improved drug delivery. Adv Drug Deliv Rev. 2017;110-111:3-12. doi:10.1016/j. addr.2016.03.008

12. Wang Y, Xie Y, Kilchrist KV, Li J, Duvall CL, Oupický D. Endosomolytic and tumor-penetrating mesoporous silica nanoparticles for siRNA/miRNA combination cancer therapy. ACS Appl Mater Interfaces. 2020;12(4):4308-4322. doi:10.1021/acsami.9b21214

13. Teesalu T, Sugahara KN, Kotamraju VR, Ruoslahti E. C-end rule peptides mediate neuropilin-1-dependent cell, vascular, and tissue penetration. Proc Natl Acad Sci U S A. 2009;106(38):16157-16162. doi:10.1073/pnas.0908201106

14. Kim D, Kang J, Wang T, et al. Two-photon in vivo imaging with porous silicon nanoparticles. Adv Mater. 2017;29:39. doi:10.1002/ adma.201703309

15. Ding N, Zou Z, Sha H, et al. iRGD synergizes with PD-1 knockout immunotherapy by enhancing lymphocyte infiltration in gastric cancer. Nat Commun. 2019;10(1):1336. doi:10.1038/s41467-01909296-6

16. Zuo H. iRGD: a promising peptide for cancer imaging and a potential therapeutic agent for various cancers. J Oncol. 2019;2019:9367845. doi:10.1155/2019/9367845

17. Fu S, Xu X, Ma Y, Zhang S, Zhang S. RGD peptide-based non-viral gene delivery vectors targeting integrin $\alpha(v) \beta(3)$ for cancer therapy. J Drug Target. 2019;27(1):1-11. doi:10.1080/1061186X.2018.1455841

18. Li M, Tang Z, Zhang D, et al. Doxorubicin-loaded polysaccharide nanoparticles suppress the growth of murine colorectal carcinoma and inhibit the metastasis of murine mammary carcinoma in rodent models. Biomaterials. 2015;51:161-172. doi:10.1016/j. biomaterials.2015.02.002

19. Alipour M, Baneshi M, Hosseinkhani S, et al. Recent progress in biomedical applications of RGD-based ligand: from precise cancer theranostics to biomaterial engineering: a systematic review. J Biomed Mater Res A. 2020;108(4):839-850. doi:10.1002/jbm.a.36862

20. Hurtado de Mendoza T, Mose ES, Botta GP, et al. Tumor-penetrating therapy for beta5 integrin-rich pancreas cancer. Nat Commun. 2021;12(1):1541. doi:10.1038/s41467-021-21858-1

21. Sun Q, Zhou Z, Qiu N, Shen Y. Rational design of cancer nanomedicine: nanoproperty integration and synchronization. Adv Mater. 2017;29:14. doi:10.1002/adma.201606628

22. Kong L, Campbell F, Kros A. DePEGylation strategies to increase cancer nanomedicine efficacy. Nanoscale Horizons. 2019;4 (2):378-387. doi:10.1039/C8NH00417J

23. Dai J, Dong X, Wang Q, Lou X, Xia F, Wang S. PEG-polymer encapsulated aggregation-induced emission nanoparticles for tumor theranostics. Adv Healthc Mater. 2021;e2101036. doi:10.1002/ adhm.202101036

24. Ishida T, Ichihara M, Wang $X$, et al. Injection of PEGylated liposomes in rats elicits PEG-specific IgM, which is responsible for rapid elimination of a second dose of PEGylated liposomes. J Control Release. 2006;112(1):15-25. doi:10.1016/j.jconrel.2006.01.005

25. Zou Y, Liu Y, Yang Z, et al. Effective and targeted human orthotopic glioblastoma xenograft therapy via a multifunctional biomimetic nanomedicine. Adv Mater. 2018;30(51):e1803717. doi:10.1002/ adma.201803717

26. Castro F, Martins C, Silveira MJ, Moura RP, Pereira CL, Sarmento B. Advances on erythrocyte-mimicking nanovehicles to overcome barriers in biological microenvironments. Adv Drug Deliv Rev. 2021;170:312-339. doi:10.1016/j.addr.2020.09.001

27. Izzati Mat Rani NN, Alzubaidi ZM, Azhari H. Novel engineering: biomimicking erythrocyte as a revolutionary platform for drugs and vaccines delivery. Eur J Pharmacol. 2021;900:174009. doi:10.1016/j. ejphar.2021.174009

28. Xia Q, Zhang Y, Li Z, Hou X, Feng N. Red blood cell membrane-camouflaged nanoparticles: a novel drug delivery system for antitumor application. Acta pharmaceutica Sinica B. 2019;9 (4):675-689. doi:10.1016/j.apsb.2019.01.011 
29. Yang Z, Gao D, Guo X, et al. Fighting immune cold and reprogramming immunosuppressive tumor microenvironment with red blood cell membrane-camouflaged nanobullets. ACS Nano. 2020;14 (12):17442-17457. doi:10.1021/acsnano.0c07721

30. Li S, Liu J, Sun M, Wang J, Wang C, Sun Y. Cell membrane-camouflaged nanocarriers for cancer diagnostic and therapeutic. Front Pharmacol. 2020;11:24. doi:10.3389/ fphar.2020.00024

31. Jo S, Kim I, Lee W, et al. Highly sensitive and wide-range nanoplasmonic detection of fibrinogen using erythrocyte membrane-blanketed nanoparticles. Biosens Bioelectron. 2019;135:216-223. doi:10.1016/j. bios.2019.04.030

32. Xie W, Deng WW, Zan M, et al. Cancer cell membrane camouflaged nanoparticles to realize starvation therapy together with checkpoint blockades for enhancing cancer therapy. ACS Nano. 2019;13 (3):2849-2857. doi:10.1021/acsnano.8b03788

33. Que X, Su J, Guo P, et al. Study on preparation, characterization and multidrug resistance reversal of red blood cell membrane-camouflaged tetrandrine-loaded PLGA nanoparticles. Drug Deliv. 2019;26 (1):199-207. doi:10.1080/10717544.2019.1573861

34. Xie X, Hu X, Li Q, et al. Unraveling cell-type-specific targeted delivery of membrane-camouflaged nanoparticles with plasmonic imaging. Nano Lett. 2020;20(7):5228-5235. doi:10.1021/acs. nanolett.0c01503

35. Nguyen TL, Choi Y, Kim J. Mesoporous silica as a versatile platform for cancer immunotherapy. Adv Mater. 2019;31(34):e1803953. doi:10.1002/adma.201803953

36. Nie D, Dai Z, Li J, et al. Cancer-cell-membrane-coated nanoparticles with a yolk-shell structure augment cancer chemotherapy. Nano Lett. 2020;20(2):936-946. doi:10.1021/acs.nanolett.9b03817

37. Jiang Q, Liu Y, Guo R, et al. Erythrocyte-cancer hybrid membrane-camouflaged melanin nanoparticles for enhancing photothermal therapy efficacy in tumors. Biomaterials. 2019;192:292-308. doi:10.1016/j.biomaterials.2018.11.021
38. Dehaini D, Wei X, Fang RH, et al. Erythrocyte-platelet hybrid membrane coating for enhanced nanoparticle functionalization. $A d v$ Mater. 2017;29(16):1606209.

39. Mao X, Liu J, Gong Z, et al. iRGD-conjugated DSPE-PEG2000 nanomicelles for targeted delivery of salinomycin for treatment of both liver cancer cells and cancer stem cells. Nanomedicine. 2015;10 (17):2677-2695. doi:10.2217/nnm.15.106

40. Zhang B, Luo Z, Liu J, Ding X, Li J, Cai K. Cytochrome c end-capped mesoporous silica nanoparticles as redox-responsive drug delivery vehicles for liver tumor-targeted triplex therapy in vitro and in vivo. $J$ Control Release. 2014;192:192-201. doi:10.1016/j.jconrel.2014.06.037

41. Qin Y, Chen W, Jiang G, et al. Interfering MSN-NONO complex-activated CREB signaling serves as a therapeutic strategy for triple-negative breast cancer. Sci Adv. 2020;6(8):eaaw9960. doi:10.1126/sciadv.aaw9960

42. Paris JL, Cabanas MV, Manzano M, Vallet-Regi M. Polymer-grafted mesoporous silica nanoparticles as ultrasound-responsive drug carriers. ACS Nano. 2015;9(11):11023-11033. doi:10.1021/acsnano.5b04378

43. Sun Y, Zhao Y, Teng S, et al. Folic acid receptor-targeted human serum albumin nanoparticle formulation of cabazitaxel for tumor therapy. Int $J$ Nanomedicine. 2019;14:135-148. doi:10.2147/IJN. S181296

44. Gao F, Zhang J, Fu C, et al. iRGD-modified lipid-polymer hybrid nanoparticles loaded with isoliquiritigenin to enhance anti-breast cancer effect and tumor-targeting ability. Int $J$ Nanomedicine. 2017;12:4147-4162. doi:10.2147/IJN.S134148

45. Gregory JV, Kadiyala P, Doherty R, et al. Systemic brain tumor delivery of synthetic protein nanoparticles for glioblastoma therapy. Nat Commun. 2020;11(1):5687. doi:10.1038/s41467-020-19225-7
International Journal of Nanomedicine

\section{Publish your work in this journal}

The International Journal of Nanomedicine is an international, peerreviewed journal focusing on the application of nanotechnology in diagnostics, therapeutics, and drug delivery systems throughout the biomedical field. This journal is indexed on PubMed Central, MedLine, CAS, SciSearch ${ }^{\mathbb{R}}$, Current Contents ${ }^{\mathbb{R}} /$ Clinical Medicine, $^{2}$
Journal Citation Reports/Science Edition, EMBase, Scopus and the Elsevier Bibliographic databases. The manuscript management system is completely online and includes a very quick and fair peer-review system, which is all easy to use. Visit http://www.dovepress.com/ testimonials.php to read real quotes from published authors. 